\title{
On the contact domain method: A comparison of penalty and Lagrange multiplier implementations
}

\author{
R. Weyler ${ }^{\text {a }}$ J. Oliver ${ }^{\text {b,* }}$, T. Sain ${ }^{\text {b }}$, J.C. Cante ${ }^{\mathrm{a}}$ \\ ${ }^{a}$ E.T.S. d'Enginyería Industrial i Aeronáutica de Terrassa, Spain \\ ${ }^{\mathrm{b}}$ E.T.S. d'Enginyers de Camins, Canals i Ports, Technical University of Catalonia (UPC), Campus Nord UPC, Mòdul C-1, c/Jordi Girona 1-3, 08034 Barcelona, Spain
}

\section{A R T I C L E I N F O}

\section{Article history:}

Received 12 April 2010

Received in revised form 27 December 2010

Accepted 12 January 2011

Available online $\mathrm{xxxx}$

\section{Keywords:}

Contact domain method

Lagrange multiplier method

Penalty method

Regularized penalty method

Interior penalty method

\begin{abstract}
A B S T R A C T
This work focuses on the assessment of the relative performance of the so-called contact domain method, using either the Lagrange multiplier or the penalty strategies. The mathematical formulation of the contact domain method and the imposition of the contact constraints using a stabilized Lagrange multiplier method are taken from the seminal work (as cited later), whereas the penalty based implementation is firstly described here. Although both methods result into equivalent formulations, except for the difference in the constraint imposition strategy, in the Lagrange multiplier method the constraints are enforced using a stabilized formulation based on an interior penalty method, which results into a different estimation of the contact forces compared to the penalty method. Several numerical examples are solved to assess certain numerical intricacies of the two implementations. The results show that both methods perform similarly as one increases the value of the penalty parameter or decreases the value of the stabilization factor (in case of the Lagrange multiplier method). However there seems to exist a clear advantage in using the Lagrange multiplier based strategy in a few critical situations, where the penalty method fails to produce convincing results due to excessive penetration.
\end{abstract}

(c) 2011 Elsevier B.V. All rights reserved.

\section{Motivation}

The computational modeling and analysis of structural contact problems have been important subjects of interest over the past several decades. Despite the significant progress achieved in the subject, it still poses a challenge in non-linear problems, especially when the aim is to develop accurate and efficient algorithms based on implicit methods.

While developing a contact formulation two main ingredients may be basically chosen:

- A scheme to discretize the contact surfaces or the interface between them.

- A technique to enforce the contact constraints.

Most of the existing contact formulations impose the contact constraints on the boundary of one of the contacting bodies, which necessitates the projection of certain quantities from one contacting surface onto the other. The most popular discretization strategy in the context of large deformation contact problems is the nodeto-segment approach developed by Hallquist et al. [7] and further extended to more general cases by Bathe and Chaudhary [2], Simo

\footnotetext{
* Corresponding author.

E-mail address: xavier.oliver@upc.edu (J. Oliver).
}

et al. [19], Wriggers and Simo [23] and Papadopoulos and Taylor [16].

The non-penetration conditions are enforced by preventing the nodes on one of the contact surface (the 'slave' one) from penetrating on the counterpart contact surface (the 'master' one). The methodology inherits some drawbacks such as: (a) failure in passing the contact patch test for a single pass algorithm [8] and (b) being prone to lock when considered as a two-pass algorithm due to the overconstraining of the contact surface. Moreover in the classical formulation, the algorithm is unable to deal with some cases where the identification of the master segment is ambiguous with respect to the slave nodes. Recently, Zavarise and De Lorenzis [26] have facilitated new techniques to overcome such problems.

In recent years other discretization schemes were also developed, based on a continuous treatment of the contact constraints. The latest segment-to-segment discretization strategies are based on the so-called mortar method initially introduced in the context of domain decomposition methods [3]. In contrast to the node-tosegment discretization, the continuity constraints are not enforced at discrete nodal points but they are formulated along the entire coupling boundary in a weak integral sense. This method is particularly well suited to exchange information of two discretized domains along common, in general non-conforming, surface grids and it provides the optimal convergence rate of the finite element solution by imposing a weak coupling between degrees of freedom. 
Due to its obvious advantages, this method has found its application in many of the recently developed frictionless and frictional contact formulation focusing on large deformation problems $[25,18,17,5]$.

Alike the discretization schemes, a variety of numerical methodologies have been proposed in the literature to deal with the contact constraints. Among them, the enforcement of the constraints using Lagrange multiplier methods, penalty methods, the augmented Lagrangian approach or the relatively new Nitsche method are the most common methodologies.

Lagrange multiplier methods introduce additional variables (the Lagrange multipliers) to enforce directly and exactly the contact constraint. Despite the obvious advantage of the exact enforcement of the constraint condition, the method poses some difficulties due to the additional effort required to solve the multipliers. In addition, the equations for the Lagrange multipliers introduce zeros in the diagonal of the system of equations, which pose additional difficulties if direct solution techniques are used. However applications of Lagrange multiplier based formulations are widespread till date and they can be found in $[5,10,20]$, to name a few.

On the other hand, penalty methods avoid the need for additional variables by introducing an approximation of the constraint condition. An additional term enters in the weak form of the governing equations, which penalizes the dissatisfaction of the constraint condition by a large positive penalty parameter. Theoretically, as the penalty parameter tends to infinity, the contact constraint is enforced exactly. Unfortunately, the resulting system of equations may become ill-conditioned as the penalty parameter increases, so the choice of an appropriate penalty parameter becomes a balancing act between accuracy and stability. Way back in 1995 [4], a consistent penalty method had been used to model metal forming process using a visco-elastic formulation. Recently, Fischer and Wriggers [6] used penalty methods for solving 2D frictional contact for large deformation problems. Along with these conventional techniques, the augmented Lagrangian method is often chosen to cope with the contact inequality constraints inasmuch as it combines the regularizing effect of the penalty method and the exact satisfaction of the constraints ensured by the Lagrange multiplier method, without having the ill-conditioning problem inherent to the former $[21,27]$. In a recent work by Wriggers and Zavarise [24], a purely displacement based formulation has been presented based on a non-standard variational formulation introduced by Nitsche. It has been found that the new discretization scheme performs better than the standard penalty formulation for frictionless contact problems.

In the present paper the recently developed method for discretizing the contact interface named as contact domain method (CDM), originally proposed in [14], and its relative performance is assessed with respect to the schemes used for imposing the contact constraints i.e. the Lagrange multiplier and the penalty strategies. As mentioned in [14], in the CDM the contact domain can be interpreted as a fictitious intermediate region that connects the potential contact surfaces and has the same dimension as the deformable contacting bodies. The utilized contact domain is discretized with a non-overlapping set of patches that leads to a pairing of the contacting entities (nodes, segments and surfaces) in the contact boundaries. Based on this discretization scheme, the geometric normal and tangential contact constraints are formulated in terms of dimensionless, strain-like measures.

Although the detailed theoretical derivations and numerical implementations are described in [14], for the relevance of the present discussion the equations for describing the CDM are reconsidered in the following sections. Then the two strategies for imposing the contact constraints (Lagrange multipliers and a regularized penalty) are described and their equivalences are found. Finally, both schemes are compared by using the patch test and a number of relevant numerical examples, and their relative performance is evaluated in terms of accuracy and robustness and some relevant conclusions are presented.

\section{Review of the contact domain method: stabilized Lagrange multiplier implementation}

The contact domain method was originally proposed for 2D contacting bodies in [14] and validated with a number of numerical examples in [9]. An extension to the frictionless 3D case was given in [11]. The method poses some specific features for modeling contact between two largely deformable bodies, namely:

- The discretization scheme allowing the contact restrictions to be applied in a manifold (the contact domain) of the same dimension as the contacting bodies, which is in contrast with other (node-to-segment, mortar etc.) methods, where normally those restrictions are imposed in a domain whose dimension is one order less than the dimension of the body,

- That defined contact domain is then discretized, and completely covered by a set of non-overlapping contact patches, which implicitly determine all possible node-to-segment pairings, this having relevant consequences as for passing the patch.

- The contact domain, covering the inter-space between the contacting bodies, is endowed with a displacement field interpolated from the contacting boundaries. This allows defining the gaps in terms of stretch-like measures, which facilitates the derivation of the gap differentiation and its linearization.

As for the type of contact patches, it is worth mentioning that, for a 2D problem, triangular or quadrilateral patches can be used for the contacting domain irrespective of the meshing pattern used for the elements. However, linear triangular shape poses little advantages as mentioned in Section 2.2 of [14]. Hence, the entire formulation is restricted to linear triangular shaped contact patches in [14] and herein.

\subsection{Contact patches and gap definitions}

Let us consider two contacting bodies $\mathcal{B}^{(\alpha)}, \alpha=1,2$ undergoing large deformations (see Fig. 1); the associated deformation maps $\boldsymbol{\varphi}_{t}^{(\alpha)}$ connect the material points $\mathbf{X}^{(\alpha)}$ at the reference configuration onto points $\mathbf{x}^{(\alpha)}$ in the current configuration.

As mentioned in [14], at the time step $\left[t_{n}, t_{n+1}\right]$, the contact domain $D_{n}$, with boundary $\partial D_{n}$ joining part of the boundaries of the contacting bodies, can be approximated by a domain $D_{n}^{l}$ partitioned in $n_{p}$ patches $D_{n}^{(p)}$ such that

$D_{n} \approx D_{n}^{l}=\bigcup_{p=1}^{n_{p}} D_{n}^{(p)}$,

the partition having the property that the patches do not overlap and $D_{n}^{l}$ converges to the contact domain $D_{n}$ as the number of patches $D_{n}^{(p)}$ and their vertices $V^{(i)}$ increase.

With reference to Fig. 2, we introduce $\mathbf{u}^{D}(\mathbf{x})$ as the pseudo incremental displacement field, at time step $\left[t_{n}, t_{n+1}\right]$ in the contact domain, conveniently interpolated from the incremental displacement at the nodes of the contacting boundaries using the linear shape functions at the contact patches; thus defining the extrapolated pseudo incremental motion of the contact domain $\phi: D_{n} \rightarrow D_{n+1}$, $\phi\left(\mathbf{x}_{n}\right) \equiv \phi^{D}\left(\mathbf{x}_{n}\right)=\mathbf{x}_{n}+\mathbf{u}^{D}\left(\mathbf{x}_{n}\right)$ as explained in [14].

We also introduce the entities $\mathbf{x}_{n}, \overline{\mathbf{x}}_{n}, \mathbf{x}_{n+1}=\phi\left(\mathbf{x}_{n}\right)$, respectively, as the position of a pseudo-material point in the contact patch at time $t_{n}$, its projection on the base-side of the contact patch 


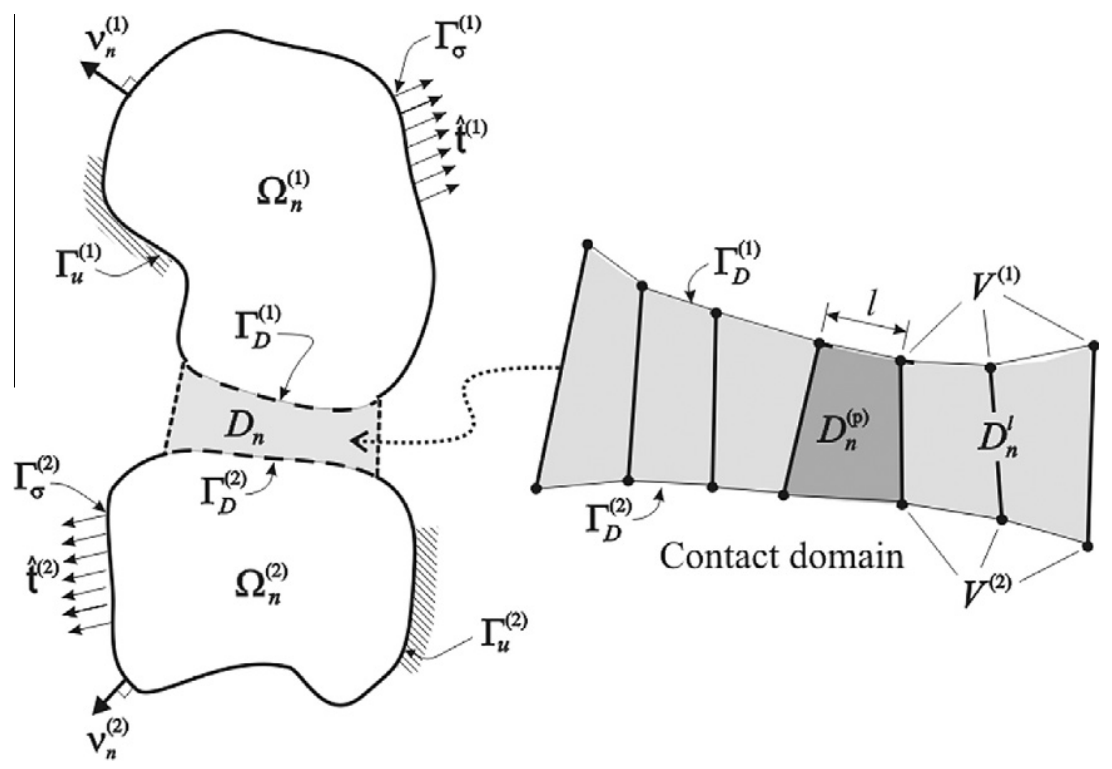

Fig. 1. Definition of the contact domain $D_{n}$ between two contacting bodies $\Omega^{(\alpha)}$ and its subdivision into patches $D_{n}^{(p)}$.

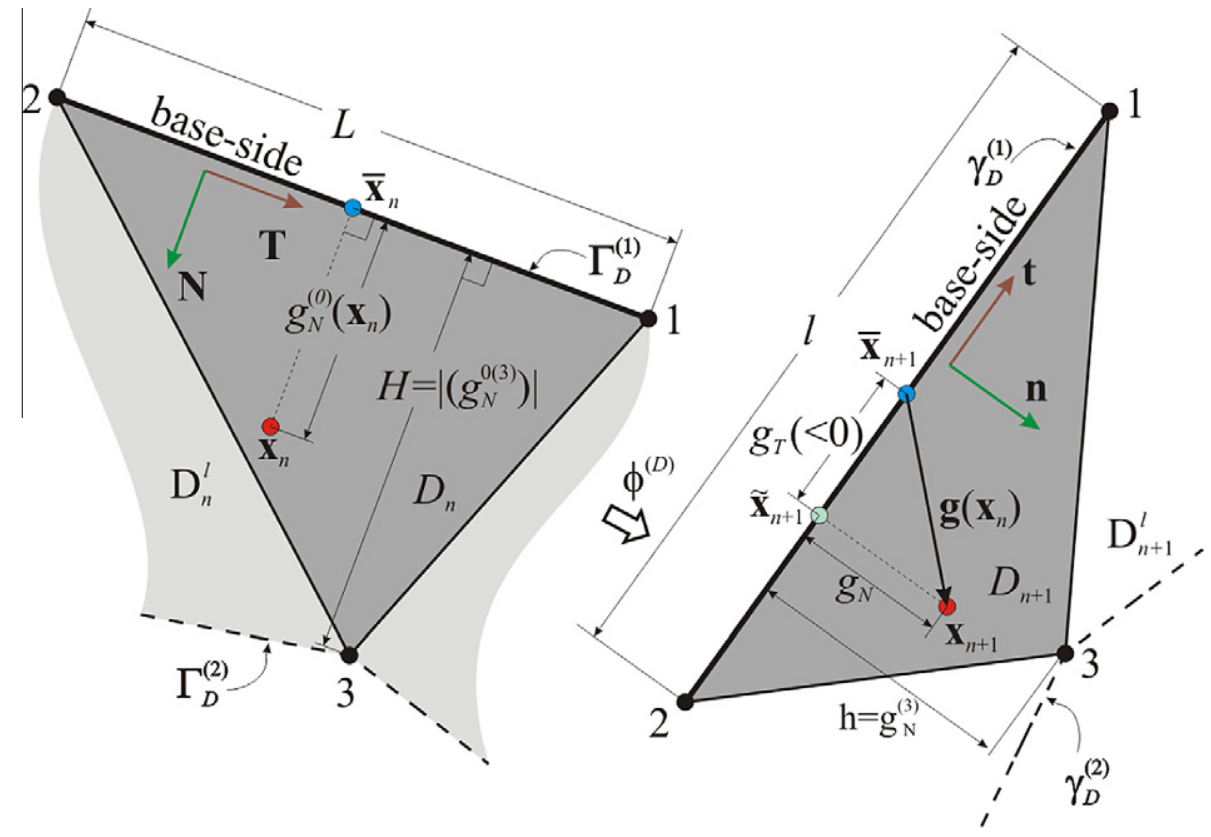

Fig. 2. Linear triangle contact patch in the previous and current configurations.

and its convected position at time $t_{n+1}$. Then, the concept of gap measures can be introduced as:

$\mathbf{g}\left(\mathbf{x}_{n}\right)=g_{N}^{(0)}\left(\mathbf{x}_{n}\right) \mathbf{f} \cdot \mathbf{N}=g_{N}(\mathbf{x}) \mathbf{n}+g_{T}(\mathbf{x}) \mathbf{t}$,

where $\mathbf{n}$ and $\mathbf{t}$ are the current normal and tangent unit vectors shown in Fig. 2. In Eq. (2) $\mathbf{f}$ is the incremental deformation gradient of $\phi$ given by:

$\mathbf{f}=G R A D\left(\phi\left(\mathbf{x}_{n}\right)\right)=\frac{\partial \mathbf{x}_{n+1}}{\partial \mathbf{x}_{n}}$.

For a detailed derivation of Eq. (2), the reader can refer to Eq. (15) of [14]. From the definition of the total gap vector, $\mathbf{g}\left(\mathbf{x}_{n}\right)$ in Eq. (2), one can further write the normal and tangential gap intensity (gap per unit of initial normal gap), in a non-dimensionalized and patch-wise constant form as [see Eqs. (16) and (17) of [14]]:

$$
\begin{aligned}
& \bar{g}_{N}=\frac{g_{N}\left(\mathbf{x}_{n}\right)}{\left|g_{N}^{(0)}\left(\mathbf{x}_{n}\right)\right|}=\operatorname{sign}\left(g_{N}^{(0)}\right) \mathbf{n} \cdot \mathbf{f} \cdot \mathbf{N}, \\
& \bar{g}_{T}=\frac{g_{T}\left(\mathbf{x}_{n}\right)}{\left|g_{N}^{(0)}\left(\mathbf{x}_{n}\right)\right|}=\operatorname{sign}\left(g_{N}^{(0)}\right) \mathbf{t} \cdot \mathbf{f} \cdot \mathbf{N},
\end{aligned}
$$

where $g_{N}^{(0)}(\cdot)$ is the initial normal gap defined for every point $\mathbf{x}_{n} \in D_{n}$ in the reference configuration and $g_{N}=\mathbf{g} \cdot \mathbf{n}$ and $g_{T}=\mathbf{g} \cdot \mathbf{t}$ are, respectively, the normal and tangential components of the physical gap vector $\mathbf{g}\left(\mathbf{x}_{n}\right)$ in the current configuration as shown in Fig. 2.

\subsection{Boundary value problem}

With the above definitions in hand, the field equations for the two contacting bodies are given as the following: 
FIND : $\mathbf{u}^{(\alpha)}\left(\mathbf{x}_{n}^{(\alpha)}\right): \Omega_{n} \rightarrow \mathbb{R}^{2}$

FULFILLING :

Momentum equation : $\rho^{(\alpha)} \ddot{u}^{(\alpha)}=\operatorname{DIV} \mathbf{P}^{(\alpha)}+\mathbf{b}^{(\alpha)}$ in $\Omega_{n}^{(\alpha)}$,

Constitutive model : $\mathbf{P}^{(\alpha)}=\Sigma^{(\alpha)}\left(\mathbf{u}^{(\alpha)}\right)$ in $\Omega_{n}^{(\alpha)}$,

Dirichlet's boundary conditions : $\mathbf{u}^{(\alpha)}=\hat{\mathbf{u}}^{(\alpha)}$ in $\Gamma_{u}^{(\alpha)}$,

Neumann's boundary conditions : $\mathbf{P}^{(\alpha)} \cdot \mathbf{N}^{(\alpha)}=\hat{\mathbf{t}}^{(\alpha)}$ in $\Gamma_{\sigma}^{(\alpha)}$.

Herein $\mathbf{P}^{(\alpha)}$ and $\mathbf{b}^{(\alpha)}$ are the first Piola-Kirchhoff stress tensor (given via an appropriate constitutive relation as in Eq. (8)) and the body forces on $\Omega^{(\alpha)}$, respectively. Furthermore, $\ddot{\mathbf{u}}^{(\alpha)}$ and $\rho^{(\alpha)}$ represent, respectively, the material acceleration field and the density of the bodies. The appropriate boundary conditions are given by the prescribed displacements $\hat{\mathbf{u}}^{(\alpha)}$ and tractions $\hat{\mathbf{t}}^{(\alpha)}$, acting on the boundaries $\Gamma_{u}^{(\alpha)}$ and $\Gamma_{\sigma}^{(\alpha)}$. Additionally, two constraint conditions arise due to the contact imposition, which have to be treated separately as follows.

\subsubsection{Treatment of the contact constraints using Lagrange multiplier} techniques

As explained with minute details in [14], the contact constraints are treated separately for the normal and tangential conditions.

2.2.1.1. Normal contact constraint. While imposing the normal contact constraint, one has to keep in mind the geometrical impenetrability condition and the negativity of the normal contact traction in the contact domain. Having this in mind, the normal contact constraints are formulated in the form of classical Karush-Kuhn-Tucker condition as follows:

$\lambda_{N} \leqslant 0, \quad \bar{g}_{N} \geqslant 0, \quad \lambda_{N} \bar{g}_{N}=0 \quad$ in $D_{n}$.

While for detail mathematical derivation it is necessary to refer to Section 3.1 of [14], the important features of Eq. (11) are described here. The normal Lagrange multiplier $\lambda_{N}\left(\mathbf{x}_{n}\right)$ is introduced, which fulfills the identification condition,

$\lambda_{N}\left(\mathbf{x}_{n}\right)=t_{N}^{(\alpha)}\left(\mathbf{x}_{n}\right) \quad \forall \mathbf{x}_{n} \in \Gamma_{D}^{(\alpha)}$,

where $t_{N}^{(\alpha)}$ represents the normal contact traction living in the contact domain boundaries $\Gamma_{D}^{(\alpha)}$. The geometrical impenetrability condition is then imposed in terms of non-dimensionalized gap intensity $\bar{g}_{N}$, which is given by Eq. (4).

2.2.1.2. Tangential contact constraint. To impose the tangential constraint a Coulomb-type friction model is used, which restricts the tangential traction to be,

$\left\|\mathbf{t}_{T}\right\| \leqslant \mu\left|t_{N}\right|$,

$\mu$ being the coefficient of friction. As before, a tangential Lagrange multiplier $\lambda_{T}\left(\mathbf{x}_{n}\right) \forall \mathbf{x}_{n} \in D_{n}^{l}$ is introduced, fulfilling

$\lambda_{T}\left(\mathbf{x}_{n}\right)=t_{T}^{(\alpha)}\left(\mathbf{x}_{n}\right) \quad \forall \mathbf{x}_{n} \in \Gamma_{D}^{(\alpha)}$.

To distinguish between the stick and slip state, the slip function is defined as

$\Phi=\left|\lambda_{T}\right|-\mu\left|\lambda_{N}\right|\left\{\begin{array}{l}<0 \rightarrow \text { stick, } \\ =0 \rightarrow \text { slip. }\end{array}\right.$

Further introducing a non-associative slip rule of the form

$\bar{g}_{T}=-\gamma \frac{\lambda_{T}}{\left|\lambda_{T}\right|}=-\gamma \operatorname{sign}\left(\lambda_{T}\right)$,

where $\bar{g}_{T}$ is the tangential gap intensity defined as given in (5) and $\gamma$ is the friction multiplier; as finally the conventional KarushKuhn-Tucker form of the tangential contact constraint is obtained, as mentioned in [14], given by $\gamma \geqslant 0, \quad \Phi \leqslant 0, \quad \gamma \Phi=0 \quad$ in $D_{n}$

Moreover, for the stick condition, one can identify $\lambda_{T}$ as the slip resistance $\mathcal{T}$ as [see Section 3.2 in [14]]:

$\lambda_{T} \equiv \mathcal{T}=\mu \operatorname{sign}\left(\lambda_{T}\right)\left|\lambda_{N}\right|=-\mu \operatorname{sign}\left(\bar{g}_{T}\right)\left|\lambda_{N}\right|$

\subsection{Weak form of the frictional contact problem}

In general, the virtual work principle or the weak form for the large deformation frictional contact problem is given by,

$\delta \Pi_{\text {mech }}(\mathbf{u} ; \delta \mathbf{u}):=\delta \Pi_{\text {int }, \text { ext }}\left(\mathbf{u}^{(\alpha)} ; \delta \mathbf{u}^{(\alpha)}\right)+\delta \Pi_{\text {cont }}=0 \quad \forall \delta \mathbf{u}^{(\alpha)} \in \mathcal{V}_{0}$,

where $\delta \mathbf{u}$ is the variation of the displacement $\mathbf{u}$ (virtual displacement) and $\delta \Pi_{\text {int,ext }}\left(\mathbf{u}^{(\alpha)} ; \delta \mathbf{u}^{(\alpha)}\right)$ is the sum of the internal and external virtual work done by the two contacting bodies

$\delta \Pi_{\text {int }, e x t}\left(\mathbf{u}^{(\alpha)} ; \delta \mathbf{u}^{(\alpha)}\right)=\delta \Pi_{\text {int }}\left(\mathbf{u}^{(\alpha)} ; \delta \mathbf{u}^{(\alpha)}\right)-\delta \Pi_{\text {ext }}\left(\delta \mathbf{u}^{(\alpha)}\right)$

with

$\delta \Pi_{\text {int }}\left(\mathbf{u}^{(\alpha)} ; \delta \mathbf{u}^{(\alpha)}\right)=\sum_{\alpha=1}^{2}\left\{\int_{\Omega_{n}^{(\alpha)}}\left(\rho^{(\alpha)} \ddot{\mathbf{u}}^{(\alpha)} \cdot \delta \mathbf{u}^{(\alpha)}+\mathbf{P}^{(\alpha)}: G R A D\left(\delta \mathbf{u}^{(\alpha)}\right)\right) d \Omega\right\}$

and

$\delta \Pi_{\text {ext }}\left(\delta \mathbf{u}^{(\alpha)}\right)=\sum_{\alpha=1}^{2}\left\{\int_{\Omega_{n}^{(\alpha)}} \mathbf{b}^{(\alpha)} \cdot \delta \mathbf{u}^{(\alpha)} d \Omega+\int_{\Gamma_{\sigma}^{(\alpha)}} \hat{\mathbf{t}}^{(\alpha)} \cdot \delta \mathbf{u}^{(\alpha)} d \Gamma\right\}$.

\subsubsection{Variational form using the Lagrange multiplier strategy}

Introducing the concepts of Lagrange multiplier, the primary unknowns in (6) that need to be solved are now extended to

FIND : $\begin{cases}\mathbf{u}^{(\alpha)}\left(\mathbf{x}_{n}^{(\alpha)}\right): & \Omega_{n} \rightarrow \mathbb{R}^{2}, \\ \lambda_{N}\left(\mathbf{x}_{n}\right): & D_{n} \rightarrow \mathbb{R}^{-}, \\ \lambda_{T}\left(\mathbf{x}_{n}\right): & D_{n} \rightarrow \mathbb{R}\end{cases}$

fulfilling Eqs. (7)-(10) and the constraint equations in hand as:

Lagrange multiplier identification $\left.\begin{array}{l}\lambda_{N}=t_{N}^{(\alpha)} \\ \lambda_{T}=t_{T}^{(\alpha)}\end{array}\right\}$ in $\Gamma_{D}^{(\alpha)}$.

Normal contact constraints $\lambda_{N} \leqslant 0, \quad \bar{g}_{N}\left(\mathbf{u}^{(D)}\right) \geqslant 0$,

$\lambda_{N} \bar{g}_{N}\left(\mathbf{u}^{(D)}\right)=0$ in $D_{n}$.

Tangential contact constraints $\gamma \geqslant 0, \quad \Phi \leqslant 0$,

$\gamma \Phi=0$ in $D_{n}$.

It has been pointed out in [14], that the contact interface changes from one time-step to the other in the case of the large deformation frictional contact problem. Hence, an active set strategy is employed to identify the present contact area and to distinguish between the stick and slip domains. According to that, the active normal contact domain and active tangential stick domain are given by,

$D_{n}^{(N)}:=\left\{\mathbf{x}_{n} \mid \lambda_{N}\left(\mathbf{x}_{n}\right)<0\right\}$

and

$D_{n}^{(T)}:=\left\{\mathbf{x}_{n} \mid \Phi\left(\mathbf{x}_{n}\right)<0\right\}$,

respectively. Finally, the identification of the active domains together with the inequality constraints as defined in Eqs. (25) and (26), transforms the original inequality constrained problem into an equality constrained system of equations as given below: 


$$
\text { FIND : } \begin{cases}\mathbf{u}^{(\alpha)}\left(\mathbf{x}_{n}^{(\alpha)}\right): & \Omega_{n} \rightarrow \mathbb{R}^{2}, \\ \lambda_{N}\left(\mathbf{x}_{n}\right): & D_{n}^{(N)} \rightarrow \mathbb{R}^{-}, \\ \lambda_{T}\left(\mathbf{x}_{n}\right): & D_{n}^{(T)} \rightarrow \mathbb{R} .\end{cases}
$$

Additionally, Eqs. (25) and (26) of the original problem now change to:

Coulomb's friction law : $\mathcal{T} \equiv \lambda_{T}$

$$
=-\mu \operatorname{sign}\left(\bar{g}_{T}\right)\left|\lambda_{N}\right| \text { in } D_{n}^{(N)} \backslash D_{n}^{(T)}
$$

Constraint conditions: $\begin{array}{ll}\bar{g}_{N}=0 & \text { in } D^{(N)}, \\ \bar{g}_{T}=0 & \text { in } D^{(T)} .\end{array}$

Now, the variational form of the above equations results into,

$$
\begin{aligned}
& \text { FIND : } \mathbf{u}^{(\alpha)} \in \mathcal{V} \text { and } \lambda=\left[\lambda_{N}, \lambda_{T}\right] \in \mathcal{L}_{N} \times \mathcal{L}_{T} \\
& \begin{aligned}
\text { FULFILLING : } \delta \Pi_{\text {mech }}(\mathbf{u}, \lambda ; \delta \mathbf{u}):= & \delta \Pi_{\text {int } \text { ext }}\left(\mathbf{u}^{(\alpha)} ; \delta \mathbf{u}^{(\alpha)}\right) \\
& +\delta \Pi_{\text {cont }}\left(\mathbf{u}^{(\alpha)}, \lambda ; \delta \mathbf{u}^{(\alpha)}\right) \\
= & 0 \quad \forall \delta \mathbf{u}^{(\alpha)} \in \mathcal{V}_{0},
\end{aligned}
\end{aligned}
$$

where $\mathcal{V}, \mathcal{L}_{N}, \mathcal{L}_{T}$, are appropriate functional spaces, and $\delta \Pi_{\text {mech }}$ stands for the variation of the total mechanical work. The mathematical expressions for those, straightforward in solid mechanics, are reported in [14] and hence avoided here. The additional term $\delta \Pi_{\text {cont }}\left(\mathbf{u}^{(\alpha)}, \lambda ; \delta \mathbf{u}^{(\alpha)}\right)$ due to the contact part is the contact virtual work of the Lagrange multipliers along the variation of the gap intensities in normal and tangential directions, and it is given by

$$
\begin{aligned}
\delta \Pi_{\text {cont }}\left(\mathbf{u}^{(\alpha)}, \lambda ; \delta \mathbf{u}^{(\alpha)}\right)= & \underbrace{\int_{D_{n}^{(N)}} \lambda_{N} \delta \bar{g}_{N}\left(\mathbf{u}^{D}\right) d D}_{\text {normal contact }}+\underbrace{\int_{D_{n}^{(T)}} \lambda_{T} \delta \bar{g}_{T}\left(\mathbf{u}^{D}\right) d D}_{\text {stick }} \\
& +\underbrace{\int_{D_{n}^{(N)} \backslash D_{n}^{(T)}} \mathcal{T} \delta \bar{g}_{T}\left(\mathbf{u}^{D}\right) d D}_{\text {slip }} .
\end{aligned}
$$

As it has been mentioned earlier, in case of the Lagrange multiplier method, the variational constraint equations are added as follows:

$\delta \Pi_{\lambda_{N}}\left(\mathbf{u}^{(D)} ; \delta \lambda_{N}\right)=\int_{D_{n}^{(N)}} \delta \lambda_{N} \bar{g}_{N}\left(\mathbf{u}^{(D)}\right) d D=0 \quad \forall \delta \lambda_{N} \in \mathcal{L}_{N}$

and

$\delta \Pi_{\lambda_{T}}\left(\mathbf{u}^{(D)} ; \delta \lambda_{T}\right)=\int_{D_{n}^{(T)}} \delta \lambda_{T} \bar{g}_{T}\left(\mathbf{u}^{(D)}\right) d D=0 \quad \forall \delta \lambda_{T} \in \mathcal{L}_{T}$.

With this variational formulation in hand, any standard Galerkin based discretization can be adopted to obtain an equivalent system of equations to be solved by standard finite element schemes. The detailed description of the FE discretization, which can be found in [14] is not the goal of the present paper and hence avoided.

2.3.1.1. Stabilization procedure: interior penalty (Nitsche's) method. As it has been stated in [14], the previous Lagrange multiplier based formulation results into possible instability/locking of the matrix equation due to zeros appearing in the diagonal of the stiffness matrix. To avoid this an interior penalty method (Nitsche method) [13] is used, which introduces a stabilization parameter $\tau$ in the variational Eqs. (35) and (36) which now read:

$$
\begin{aligned}
\delta & \Pi_{\lambda_{N}}\left(\mathbf{u}, \lambda_{N} ; \delta \lambda_{N}\right)=\int_{D_{n}^{(N)}} \delta \lambda_{N} \bar{g}_{N}\left(\mathbf{u}^{(D)}\right) d D \\
+ & \underbrace{\int_{\partial D_{n}^{(N)} \cap \Gamma_{D}^{(\alpha)}} \delta \lambda_{N} \tau\left(t_{N}\left(\mathbf{u}^{(\alpha)}\right)-\lambda_{N}\right) d \Gamma}_{\text {additional term }}=0 \quad \forall \delta \lambda_{N} \in \mathcal{L}_{N}
\end{aligned}
$$

and

$$
\begin{aligned}
& \delta \Pi_{\lambda_{T}}\left(\mathbf{u}, \lambda_{T} ; \delta \lambda_{T}\right)=\int_{D_{n}^{(T)}} \delta \lambda_{T} \bar{g}_{T}\left(\mathbf{u}^{(D)}\right) d D \\
& +\underbrace{\int_{\partial D_{n}^{(T)} \cap \Gamma_{D}^{(\alpha)}} \delta \lambda_{T} \tau\left(t_{T}\left(\mathbf{u}^{(\alpha)}\right)-\lambda_{T}\right) d \Gamma}_{\text {additional term }}=0 \quad \forall \delta \lambda_{T} \in \mathcal{L}_{T} .
\end{aligned}
$$

It has to be noted that a mathematical proof for the stability condition for the Nitsche method in the case of small strains has been reported in [12].

Remark 2.1. The point to be noted here is that, a careful observation of the previous equations may give an impression that a penalty term in the form of stabilization factor $\tau$ is applied. However, it should be made clear at this point, that the stabilization parameter does not play the role of a classical penalty factor. The additional terms arising due to the inclusion of $\tau$ essentially vanish with mesh refinement. Hence, no alteration persists in the variational formulation at the end. Moreover, the term $\tau$ can be made very small without affecting, necessarily, the quality of the solution, which is also against the regular role of the conventional penalty parameter. In fact, a sequence of analyses using a fixed value of $\tau$ with continuous refinement of the finite element mesh shows the exact fulfillment of the contact constraints (see Section 5.2.2).

\section{A penalty implementation of the contact domain method}

\subsection{Variational form using a (regularized) penalty method}

For the penalty strategy, the contribution to the virtual work due to contact can be written as follows:

$$
\begin{aligned}
\delta \Pi_{\text {cont }}\left(\mathbf{u}^{(\alpha)} ; \delta \mathbf{u}^{(\alpha)}\right)= & \underbrace{\int_{D_{n}^{N}} \tilde{t}_{N}\left(\bar{g}_{N}\right) \delta \bar{g}_{N}}_{\text {normal contact }} d D+\underbrace{\int_{D_{n}^{T}} \tilde{t}_{T}\left(\bar{g}_{T}\right) \delta \bar{g}_{T}}_{\text {stick }} d D \\
& +\underbrace{\int_{D_{n}^{N} / D_{n}^{T}} \tilde{\mathcal{T}} \delta \bar{g}_{T}}_{\text {slip }} d D \quad \forall \delta \mathbf{u}^{(\alpha)} \in \mathcal{V}_{0} .
\end{aligned}
$$

Comparing Eq. (39) with (34) it can be noticed that, in the case of the penalty method the contact term is a function of the penalized tractions $\tilde{t}_{N}$ and $\tilde{t}_{T}$, which is different from the Lagrange multiplier method, the latter method is a function of the Lagrange multipliers, which in turn are equal to the actual traction components $t_{N}$ and $t_{T}$. To compute the contact tractions, the constraint conditions are treated in a different way in the penalty strategy as described below.

\subsection{Treatment of the normal contact constraints}

Splitting the constraint conditions into the normal and tangential counterparts, for the normal contact condition, the contact force is expressed as a function of the normal gap intensity in Eq. (4) as:

$\tilde{t}_{N}=K_{N}^{*} \bar{g}_{N}$,

where $K_{N}^{*}$ is, from now on, termed the regularized normal penalty parameter. Eq. (40) can now be rewritten in terms of the normal component of the physical gap in Eq. (2) as ${ }^{1}$ :

$\tilde{t}_{N}=K_{N}^{*} \bar{g}_{N}=K_{N}^{*} \frac{g_{N}^{(3)}}{\left|g_{N}^{0(3)}\right|}=K_{N} g_{N}^{(3)}$,

\footnotetext{
${ }^{1}$ As stated before, for the considered linear contact patch the gap intensities are constant and, therefore, Eq. (4) can be stated for "any" point of the patch and the same value of $\bar{g}_{N}$.
} 
where $K_{N}$ is the physical normal penalty parameter and $g_{N}^{(3)}$ is the normal component of the physical gap corresponding to node " 3 " in Fig. 2 (the classical node-to-segment gap). Eq. (41) links the contact condition (40) to the classical node-to-segment penalty condition and establishes the relation between the regularized, $K_{N}^{*}$, and the physical, $K_{N}$, penalties:

$K_{N}^{*}=\left|g_{N}^{0(3)}\right| K_{N}=H K_{N}$,

where $g_{N}^{0(3)}(=H)$ is the initial normal gap in the reference configuration (the height of the contact patch with respect to the base-line) as shown in Fig. 2.

Now the normal constraint condition (40) can be formalized in terms of the regularized penalty parameter as:

$\tilde{t}_{N}=K_{N}^{*} \bar{g}$,

$K_{N}^{*}= \begin{cases}0 & \text { if } \bar{g}_{N} \geqslant 0 \text { (no contact), } \\ H K_{N} & \text { if } \bar{g}_{N}<0 \text { (penetration). }\end{cases}$

\subsection{Treatment of the tangential contact constraints: friction model}

For the tangential contact constraint, a Coulomb-type friction model is proposed as earlier. To maintain an analogy with a plasticity-like formulation the equation for computing the tangential contact stress is expressed in rate form as a function of the incremental slip intensity rate as:

$\dot{\tilde{t}}_{T}=K_{T}^{*}\left(\dot{\bar{g}}_{T}-\dot{\bar{g}}_{T}^{s l i p}\right)$,

where $K_{T}^{*}$ represents the regularized tangential penalty parameter. Similar to $\tilde{t}_{N}, \tilde{t}_{T}$ is also a penalized term expressed as a function of the penalized gap and hence differs from the actual $t_{T}$.

Again, from Eq. (5) the above equation can be rewritten in terms of the physical tangential gap as

$\dot{\tilde{t}}_{T}=\frac{K_{T}^{*}}{\left|g_{N}^{0(3)}\right|}\left(\dot{g}_{T}-\dot{g}_{T}^{s l i p}\right)=K_{T}\left(\dot{g}_{T}-\dot{g}_{T}^{s l i p}\right)$,

$K_{T}^{*}=K_{T}^{H}$.

In Eq. (45) the physical, $K_{T}$, and the regularized, $K_{T}^{*}$, tangential penalty parameters are related as earlier. To distinguish between the stickslip conditions the slip function is introduced as:

$\Phi\left(\tilde{t}_{T}\right)=\left|\tilde{t}_{T}\right|-\mu\left|\tilde{t}_{N}\right| \leqslant 0$.

Further, the slip rule can be written as:

$\dot{\bar{g}}_{T}^{\operatorname{slip}}=\dot{\gamma} \operatorname{sign}\left(\tilde{t}_{T}\right)$.

Now, the tangential contact conditions can be summarized in the form of the Kuhn-Tucker condition as follows:

$\dot{\gamma} \geqslant 0 ; \quad \Phi \leqslant 0 ; \quad \dot{\gamma} \Phi=0$.

\subsection{Numerical implementation of the friction model}

To implement the proposed friction model in terms of the tangential contact in a finite element scheme, one needs to compute the tangential stress $\tilde{t}_{T}$ by integrating the model in Eqs. (44)-(48). Assuming that the tangential stress, $\tilde{t}_{T, n}$, at the previous configuration $\left(t=t_{n}\right)$, is known, and since the tangential gap and the slip are zero in that configuration, the update of $\tilde{t}_{T}$ at time step $n+1$, can be made from Eq. (44) as:

$\tilde{t}_{T, n+1}=\tilde{t}_{T, n}+K_{T}^{*}\left(\bar{g}_{T}-\bar{g}_{T}^{s l i p}\right)$.

To distinguish between the stick-slip conditions one has to check the yield condition and compute the slip rate accordingly. To per- form the calculation, a trial stress state is defined at the current configuration, assuming the plastic part of the tangential slip to be zero as:

$\tilde{t}_{T, n+1}^{T R}=\tilde{t}_{T, n}+K_{T}^{*} \bar{g}_{T}$.

The actual tangential stress can now be expressed in terms of the trial state as follows:

$\tilde{t}_{T, n+1}=\tilde{t}_{T, n+1}^{T R}-K_{T}^{*} \Delta \gamma \operatorname{sign}\left(\tilde{t}_{T, n+1}\right)$.

From the above expressions, it can easily be shown that,

$\operatorname{sign}\left(\tilde{t}_{T, n+1}\right)=\operatorname{sign}\left(\tilde{t}_{T, n+1}^{T R}\right) ; \quad\left|\tilde{t}_{T, n+1}\right|=\left|\tilde{t}_{T, n+1}^{T R}\right|-K_{T}^{*} \Delta \gamma$.

The slip function (46) in terms of the trial stress can be rewritten as

$\Phi^{T R}\left(\tilde{t}_{T, n+1}^{T R}\right)=\left|\tilde{t}_{T, n+1}^{T R}\right|-\mu\left|\tilde{t}_{N, n+1}\right|$.

Finally, the tangential constraint condition can be established in terms of $\Phi^{T R}$ as:

$$
\begin{aligned}
& \Phi^{T R} \leqslant 0 ; \quad \Delta \gamma=0 ; \quad \Rightarrow \text { stick condition; } \quad \tilde{t}_{T, n+1}=\tilde{t}_{T, n+1}^{T R}, \\
& \Phi^{T R}>0 ; \quad \Delta \gamma=\frac{\Phi^{T R}}{K_{T}^{*}} ; \quad \Rightarrow \text { slip condition; } \\
& \tilde{t}_{T, n+1}=\tilde{\mathcal{T}}=\mu\left|\tilde{t}_{N, n+1}\right| \operatorname{sign}\left(\tilde{t}_{T, n+1}^{T R}\right) .
\end{aligned}
$$

Once the constraint conditions are formalized, the traction components appearing in the variational form in Eq. (39) will be known, and hence subsequently the matrix form of the system of equations can be obtained in a straightforward manner. Since the finite element implementation of the proposed problem is a textbook matter, its development is avoided here. Instead, a detail comparison between the Lagrange multiplier based implementation and the penalty strategy has been made in the final mathematical derivation, to discuss the difference or relative equivalence between them.

\section{Equivalence between Lagrange multiplier and penalty based approaches}

To point out the equivalence between the two methods derived in the previous sections, we start from the variational constraint equations for the Lagrange multiplier method (SLMM) as given in (37) and (38), and in (39) for the penalty method. For conciseness in the derivation, let us focus on the normal constraint part, as the others constraint conditions can be treated in a similar way. For a linear triangular contact patch, the integration of Eq. (37) results into a simplified form such as:

$\frac{1}{2} H \bar{g}_{N}+\tau\left(t_{N}-\Lambda_{N}\right)=0$,

where $H$ is the absolute value of the initial normal gap of node ' 3 ' and $\Lambda_{N}$ is the value of the Lagrange multiplier assumed constant in the patch. To derive the above form, the gap intensity $\bar{g}_{N}$ and the stabilization parameter $\tau$ are considered to be constant for a triangular patch. The details of the derivation can be found in Section 5.2 of [14]. Introducing a new term as the numerical gap $g_{N}^{\text {num }}$, and replacing $\bar{g}_{N}=g_{N}^{(3)} / H$, one could get,

$g_{N}^{\text {num }} \equiv g_{N}^{(3)}+2 \tau\left(t_{N}-\Lambda_{N}\right)=0$

this stating the role of $g_{N}^{n u m}$ as the actual gap numerically imposed to be zero in the formulation. Hence, using (56), $\Lambda_{N}$ can be solved as:

$\Lambda_{N}=t_{N}+\frac{1}{2 \tau} g_{N}^{(3)}$.

In case of the penalty approach, the Lagrange multiplier is replaced with the penalized traction $\tilde{t}_{N}$ (see Eq. (41)). Hence, in the penalty method, $\Lambda_{N}\left(\approx \tilde{t}_{N}\right)$ can be solved for a specific patch as: 
$\Lambda_{N} \approx \tilde{t}_{N}=K_{N} g_{N}^{(3)}$.

For a better understanding the two Eqs. (57) and (58) are plotted graphically as shown in Fig. 3.

It is seen that computation of the contact stresses as a function of the normal physical gap, $g_{N}$, is very similar in the two methods; the difference lies in the use of $\frac{1}{2 \tau}$ in the SLMM instead of the penalty parameter $K_{N}$ in the penalty method. Further, in SLMM, the straight-line starts with an intercept of $t_{N}$ which approaches to $\Lambda_{N}$ with FE mesh refinement (see Eq. (24)). Hence, a comparison can be made between the SLMM and the penalty method considering these two factors as influencing parameters on the accurate implementation of the contact constraints. For elaborative understanding of this point, the performance of the two methods has been assessed considering several numerical examples in the following section.

In case of SLMM, the exact imposition of the contact constraint is perturbed due to the use of a small non-zero value of $\tau$, which introduces an additional term in the variational equation as seen in Eq. (37). This results, also, into some additional terms in the stiffness matrices corresponding to the Lagrange multiplier method, when compared to the penalty method.

More specifically: the normal contact force vector for a linear triangular contact patch corresponding to the penalty method and Lagrange multiplier approach can be respectively written considered as (see the Appendix A):

$F_{\text {cont }}^{N(\text { penalty })}=\frac{1}{2} L\left(h K_{N}\right) \mathbf{n} \cdot \sum_{I=1}^{3}\left(h \frac{\partial N_{I}}{\partial n}\right)$

and

$F_{\text {cont }}^{N(\text { SLMM })}=\frac{1}{2} L \Lambda_{N} \mathbf{n} \cdot \sum_{I=1}^{3}\left(h \frac{\partial N_{I}}{\partial n}\right)$.

In these equations, $L$ is the length of the base vector in the reference configuration, and $h\left(=g_{N}^{(3)}\right)$ is the height of the "slave" node '3' with respect to the base vector in the current configuration, as shown in Fig. 2. Eqs. (59) and (60) prove to be the same if the equivalency $\Lambda_{N}=h K_{N}$ is made (see Eq. (58)).

Let us, now, consider the normal contact stiffness matrix for the penalty method and the Lagrange multiplier approach as obtained in the Appendix. They read

$$
\begin{aligned}
K_{i p j q}^{\text {(penalty) }} & =\frac{L}{2} K_{N}\left[\left(n_{i} h \frac{\partial N_{P}}{\partial n}\right)\left(n_{j} h \frac{\partial N_{Q}}{\partial n}\right)-h\left(t_{i} h \frac{\partial N_{P}}{\partial n}\right)\left(n_{j} \frac{\partial N_{Q}}{\partial n}\right)\right. \\
& \left.=-h\left(n_{i} \frac{\partial N_{P}}{\partial n}\right)\left(t_{j} h \frac{\partial N_{Q}}{\partial n}\right)-h^{2}\left(n_{i} \frac{\partial N_{P}}{\partial n}\right)\left(n_{j} \frac{\partial N_{Q}}{\partial n}\right)\right]
\end{aligned}
$$

and

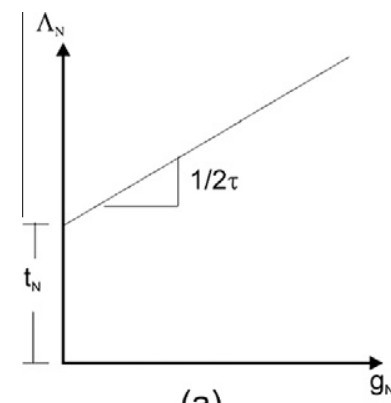

(a)

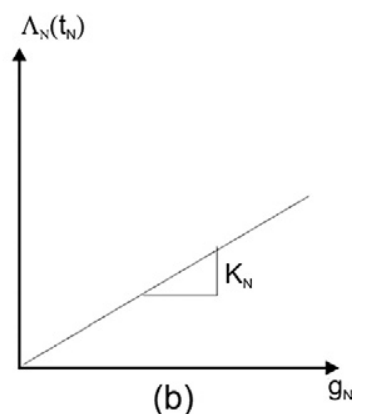

(b)
Fig. 3. Graphical representation of the relation for computing the contact stress: (a) stabilized Lagrange multiplier method; (b) penalty approach.

$$
\begin{aligned}
K_{i p j q}^{\mathrm{SLMM}}= & {\left[K_{I}\right]_{i p j q}+\left[K_{I I}\right]_{i p j q}, } \\
{\left[K_{I}\right]_{i p j q}=} & \frac{L}{2}\left[-t_{T}\left(n_{i} h \frac{\partial N_{P}}{\partial n}\right)\left(n_{j} \frac{\partial N_{Q}}{\partial n}\right)+\frac{1}{2 \tau}\left(n_{i} h \frac{\partial N_{P}}{\partial n}\right)\left(n_{j} h \frac{\partial N_{Q}}{\partial n}\right)\right. \\
& -\Lambda_{N}\left(t_{i} h \frac{\partial N_{P}}{\partial n}\right)\left(n_{j} \frac{\partial N_{Q}}{\partial t}\right) \\
& \left.-\Lambda_{N} h\left(n_{i} \frac{\partial N_{P}}{\partial t}\right)\left(n_{j} \frac{\partial N_{Q}}{\partial t}\right)-\Lambda_{N}\left(n_{i} \frac{\partial N_{P}}{\partial t}\right)\left(t_{j} h \frac{\partial N_{Q}}{\partial n}\right)\right], \\
{\left[K_{I I}\right]_{i p j q}=} & \frac{L}{2}\left(n_{i} h \frac{\partial N_{P}}{\partial n}\right)\left(n_{k} \Delta P_{k l q j} N_{l}\right),
\end{aligned}
$$

respectively, where in Eq. (62) $)_{3} \mathbf{P}$ represents the first Piola-Kirchoff stress tensor.

Assuming from Fig. 3, the fact that $K_{N}$ and $\frac{1}{2 \tau}$ are equivalent, it can be seen in Eqs. (61) and (62) that the normal stiffness matrix for SLMM contains all the terms of the penalty equations along with few additional terms arising from the body stress component at the contacting boundary.

Similar to the normal components, the equivalence between the two methods for imposing the contact constraints can be brought out for the tangential stick and slip part. For clarification purposes, detailed derivations are incorporated in the Appendix.

Remark 4.1. In spite of the shown equivalences between the Lagrange multiplier and penalty strategies, they are essentially different in terms of the constraint application. In the case of SLMM, the constraint Eqs. (37) and (38) have an additional term due to the introduction of the stabilization parameter, which in turn introduces differences in the stiffness matrix expressions. However, with mesh-refinement one can approach the exact imposition of the constraints irrespective of the value of $\tau$.

On the other hand, in the case of the penalty method, as the penalty parameters $K_{N}, K_{T}$, increase, the contact constraints are applied more accurately and the solutions should converge to those obtained using SLMM. However, as pointed out in the introduction, large values of the penalty parameter lead to an illconditioned matrix. On the other hand for smaller values of penalty parameters the contact constraint is not exactly satisfied. Therefore, the appropriate balance of these two facts, when choosing the value of the penalty parameters, has to be considered.

\section{Representative numerical simulations}

\subsection{Contact patch test}

To assess the implementations of the proposed contact algorithms, i.e. whether they are able to exactly transmit constant stress field from one body to another along an arbitrary non-conforming contact surface, a patch test has to be considered. Different patch test setups have been proposed in the literature and the one proposed by Asghar and Lyons [1] recently has been chosen here. According to it, linear displacement fields are assumed for the horizontal and vertical components $u$ and $v$ in a perfectly tied contact situation given by:

$u=(1+2 x+y) / 1000 ; \quad v=(3+5 x+7 y) / 1000$,

respectively. Obviously, the prescribed displacement field will generate constant strains in both directions and for a linear elastic material one should obtain constant stresses inside the element patch. In particular, for the considered 2D case, this chosen displacement field will generate all the stress components $\boldsymbol{\sigma}_{x x}, \boldsymbol{\sigma}_{y y}$ and $\tau_{x y}$ within a patch. To demonstrate the most critical situation, the patch test is performed considering an element patch consisting of an irregular (non-structured) non-conforming element mesh 
with an inclined contact surface as shown in Fig. 4a. To ensure a perfectly tied situation, a reasonably high friction coefficient of 1.5 is chosen for both Lagrange multiplier and penalty based analyses. For the Lagrange multiplier strategy, the minimum allowable value for the stabilization parameter $\tau$, to preclude round off errors, is observed to be $10^{-12}$. Similarly, the highest possible penalty parameter is fixed at $10^{8}$ to achieve the same order of accuracy. It has been observed that, corresponding to these parameters, the patch test, for the above mentioned element patch, is passed within machine error accuracy $\left(10^{-12}\right)$ for both the Lagrange multiplier and the penalty based implementations. In Fig. $4 \mathrm{~b}$ the difference between the maximum and minimum stress value obtained for the element patch and for all the three stress components correspond-

(a)

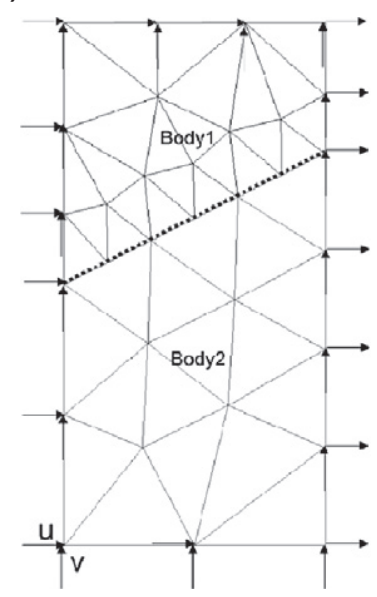

(b)

\begin{tabular}{|l|l|l|l|}
\cline { 2 - 4 } \multicolumn{1}{c|}{} & $\sigma_{x x}$ & $\sigma_{y y}$ & $\tau_{x y}$ \\
\hline SLMM & $10^{-13}$ & $10^{-13}$ & $10^{-13}$ \\
\hline Penalty & $10^{-13}$ & $10^{-13}$ & $10^{-13}$ \\
\hline
\end{tabular}

Fig. 4. Patch test with an inclined contact surface: (a) mesh geometry with the boundary condition; (b) difference between the maximum and minimum stress value. ing to the stabilized Lagrange multiplier (SLLM) and penalty methods is presented.

\subsection{Numerical examples}

\subsubsection{Compression of two blocks with non-matching surface}

Let us first consider a simple example, (as analyzed in [26]), where a rectangular block is pressed against a second block having a concave upper surface (see. Fig. 5). The lower block is restrained against vertical movement at its bottom surface, whereas the upper one is subjected to an imposed vertical downward displacement at its upper surface. The blocks are made of the same material having an elastic modulus $E=10^{6} \mathrm{~N} / \mathrm{mm}^{2}$ and a Poisson's coefficient $v=0.0$ and the contact is assumed frictionless. A large strain Neo-Hookean constitutive model is chosen. To compare the performance of the Lagrange multiplier approach with respect to the regularized penalty method, equivalence is considered between the penalty parameter $K_{N}$ and $\frac{1}{2 \tau}$ (see Section 4). Hence in the Lagrange multiplier analysis the $\tau$ values are chosen as $5 \times$ $10^{-7} \mathrm{~mm}^{3} / \mathrm{N}, 5 \times 10^{-8} \mathrm{~mm}^{3} / \mathrm{N}$ and $5 \times 10^{-9} \mathrm{~mm}^{3} / \mathrm{N}$ and the corresponding penalty values $K_{N}$ are taken as $10^{+6} \mathrm{~N} / \mathrm{mm}^{3}, 10^{+7} \mathrm{~N} / \mathrm{mm}^{3}$ and $10^{+9} \mathrm{~N} / \mathrm{mm}^{3}$. The tolerance limit is set to be $10^{-6}$ (in terms of the residual force norm) for the FE solution to converge. Fig. 6 shows the allowable displacement of the upper block, comparing the Lagrange multiplier based strategy and the penalty approach. It is seen from the graph that using the Lagrange multiplier approach, one can push the upper block up to a displacement of 1.0 $\mathrm{mm}$ for all $\tau$ values whereas the penalty method allows the complete movement only for penalty parameter $K_{N}=10^{+9} \mathrm{~N} / \mathrm{mm}^{3}$. Additionally, the solution in terms of the gap is best maintained in the Lagrange multiplier approach for either of the equivalent cases. Hence, it can be noted that, for a simple example like the above mentioned one, and in a frictionless situation, once the equivalence is considered between the two parameters of the Lagrange multiplier and regularized penalty approaches, the (a)

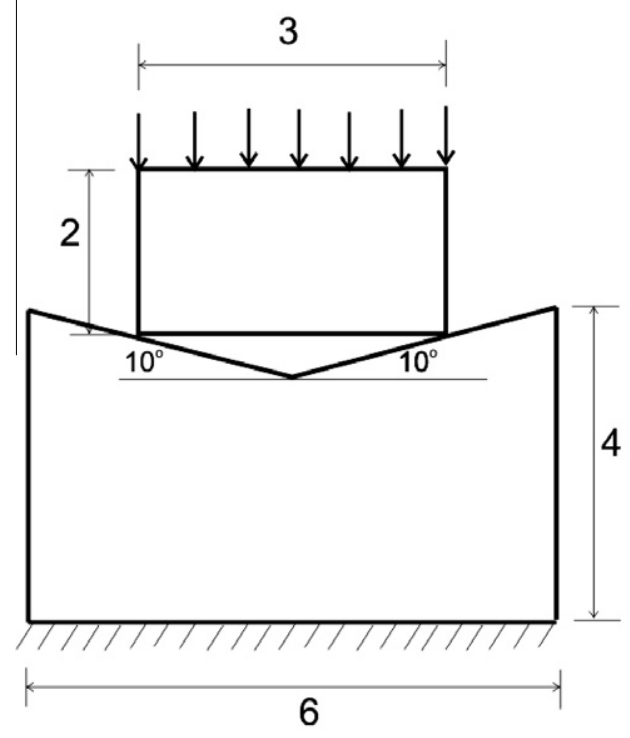

(b)

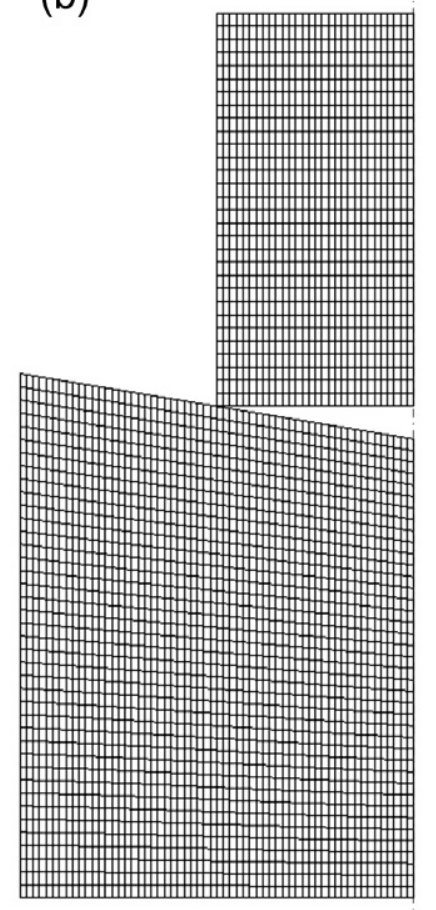

Fig. 5. (a) Geometry of the two blocks; (b) FE mesh for the blocks (exploiting the problem symmetry). 
(a)

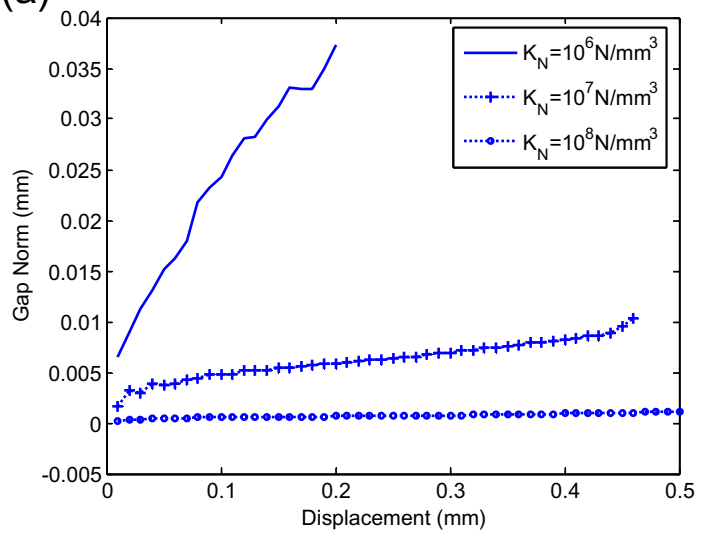

(b)

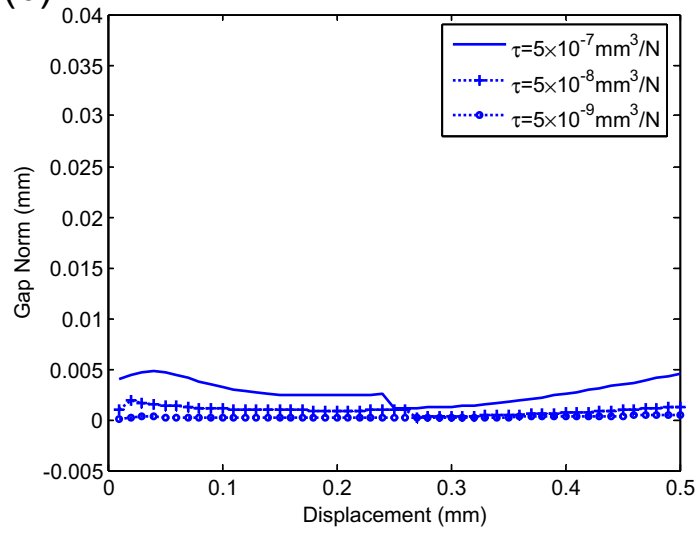

Fig. 6. Comparison of the mean gap: (a) for penalty method at different $K_{N}$ values; (b) for stabilized Lagrange multiplier for different $\tau_{N}$.

Lagrange multiplier method performs more robustly in comparison with the penalty method.

\subsubsection{Extrusion/expansion of a block}

In many industrial forming processes, it becomes important to analyze the ejection phase (i.e. separation of the two bodies in contact, which normally takes place elastically), for which the contact algorithm performance is crucial at this stage. For this purpose, we propose the test described in Fig. 7, which analyzes the effect of extrusion and expansion of a block comprising an elastic material that is sliding without friction between two vises of variable crosssection. The test consists of three stages, firstly an area of extrusion for which the geometric dimension and the mesh are adopted as described in [15], further continuing with a flat zone, followed by a tapered opening. The reference element size of the vise in the longitudinal direction is considered as $20 \mathrm{~mm}$, while the initial element size of the block is $10 \mathrm{~mm}$. As in [15], the two bodies are deformed, considering the vise device much more rigid than the block, and also restricting the movement of the outside of the vise and their ends. The example is solved in a plane strain situation, imposing a prescribed displacement of $1600 \mathrm{~mm}$ in the longitudinal base of the block. During the first part, there is a large contraction of the block in the transverse direction, which then slides without changing its deformation and finally expands to recover its original form as described in Fig. 8. Both the block and the vise consist of Neo-Hookean material, with the vise being much stiffer, with Lame coefficients, $\lambda=\mu=4 \times 10^{4} \mathrm{~N} / \mathrm{mm}^{2}$ for the block and $\lambda=\mu=4 \times 10^{7} \mathrm{~N} / \mathrm{mm}^{2}$ for the tube.

Fig. 9(a) and (b) show the horizontal reaction acting on the block as it is displaced, with the stabilized Lagrange multiplier method and with the regularized penalty method. Results correspond to an element size of $20 \mathrm{~mm}$ and different values of the penalty parameter, $K_{N}$, and stabilization parameter $\tau$.

There it can be checked that the Lagrange multiplier method shows robust convergence, to a very accurate solution for a wide range $\left(\tau \in\left[1 \times 10^{-5}, 5 \times 10^{-7}\right]\right)$ of values of the stabilization parameter. In contrast, a greater sensitivity is observed for the penalty method where convergence towards the exact solution is

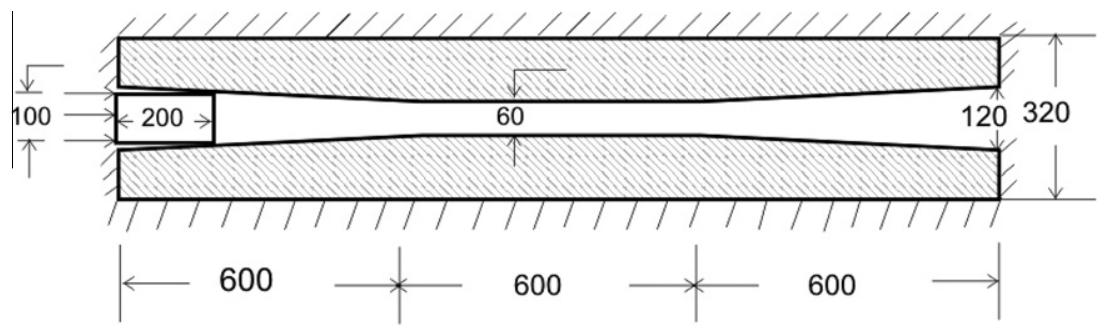

Fig. 7. Geometry for the extrusion/expansion problem (undeformed configuration, all in $\mathrm{mm}$ ).

(a)

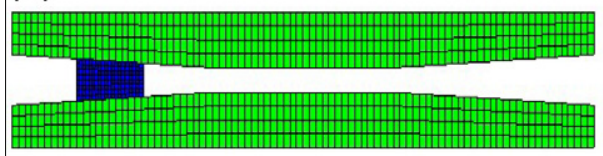

(c)

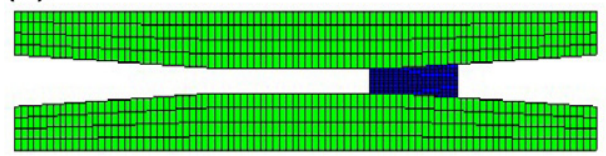

(b)

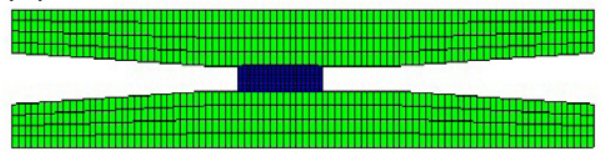

(d)

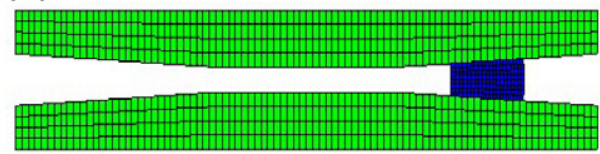

Fig. 8. (a)-(d) Deformed mesh sequence for the extrusion/expansion problem. 
(a)

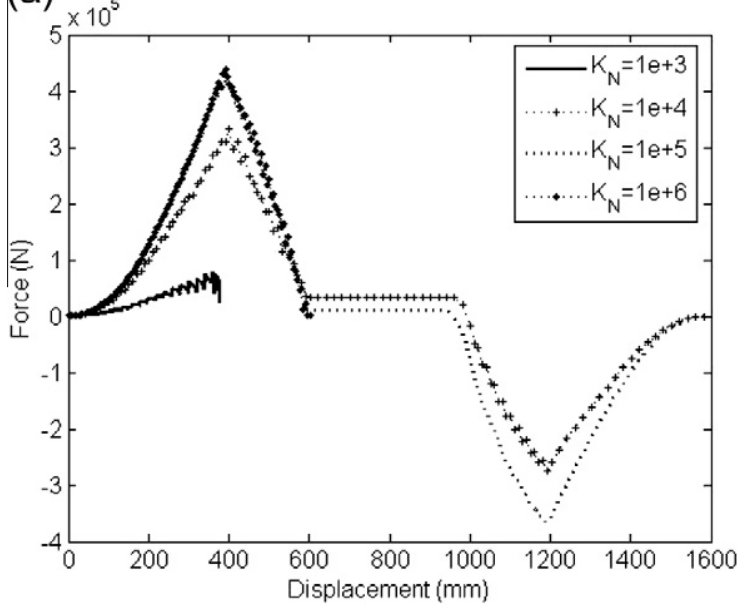

(b)

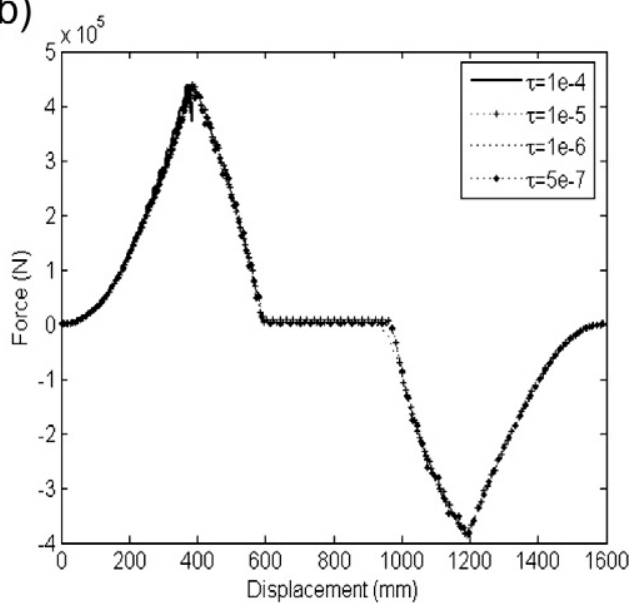

Fig. 9. Longitudinal force for $20 \mathrm{~mm}$ element size: (a) penalty method; (b) stabilized Lagrange multiplier method.

relatively slow. However, it proves unable to go beyond the extrusion state for very large penalty parameter values $\left(K_{N}=1 \times 10^{6}\right)$. Thus, the correct selection of the right penalty value becomes crucial for obtaining a robust and accurate solution.

Further, Fig. 10 compares the results with each method considering two different element sizes ( $20 \mathrm{~mm}$ and $2 \mathrm{~mm}$, respectively), showing a little dependence, for this problem, of the obtained reaction estimated by both methods with the mesh size.

Fig. 11(a) and (b) show the mean gap variable for the two methods, which is defined as [22]:

mean gap $=\sqrt{\frac{1}{n_{c}} \sum^{n_{c}} g_{N}^{2}}$,

$n_{c}$ being the number of active contact patches (notice that active patches could include patches with positive gap (or not penetrated) for the Lagrange multiplier method).

This graph shows the gap approximation of the contact condition for both the methods. From this graph one can see that using a constant $\tau$ stabilization parameter in the Lagrange multiplier approach, it is possible to improve the gap solution only with mesh refinement. However, this is not the case for the penalty method, where the mean gap increases with the mesh refinement. The increase in the mean gap values in the penalty method could be explained due to an inappropriate definition of the mean gap which is used to compare different mesh sizes.

(a)

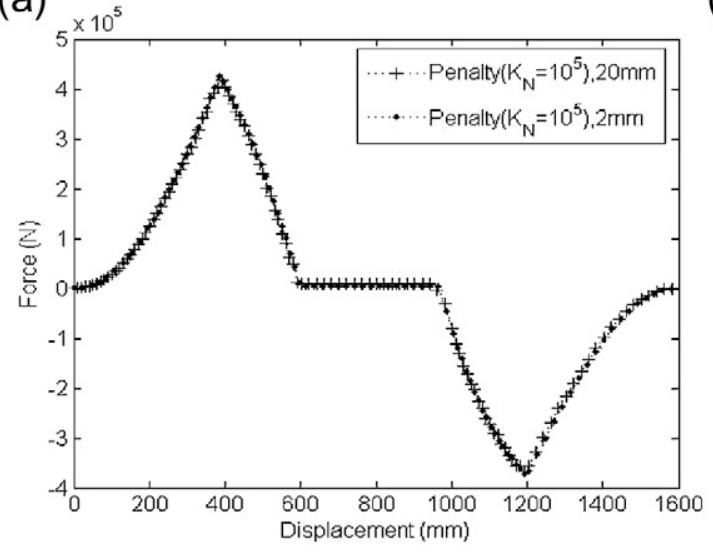

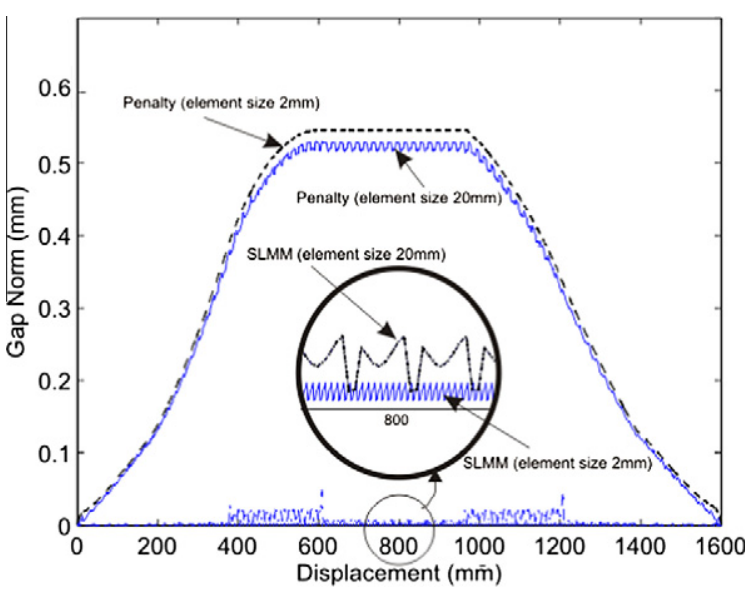

Fig. 11. Comparison of the mean gap for the penalty method at $K_{N}=10^{5} \mathrm{~N} / \mathrm{mm}^{3}$ and for the stabilized Lagrange multiplier method at $\tau_{N}=10^{-6} \mathrm{~mm}^{3} / \mathrm{N}$.

Both the methods, in the case of a coarse mesh, show undesirable oscillations during the transit along the corners of the block, which disappear completely with mesh refinement. However some oscillations still appear near the plain section of the die in case of the penalty method, which originates due to the definition of an (b)

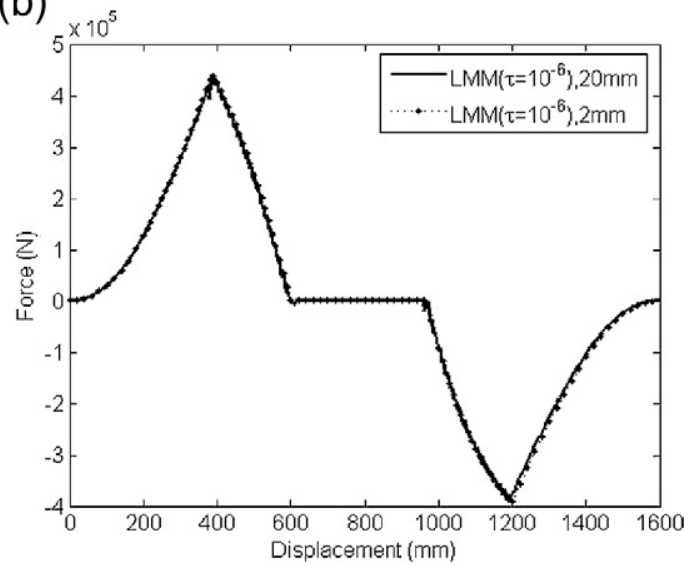

Fig. 10. Comparison of longitudinal force for $20 \mathrm{~mm}$ and $2 \mathrm{~mm}$ element size: (a) penalty method; (b) stabilized Lagrange multiplier. 
active set with excessive penetration. When the penalty parameters are extremely small, the normal calculations are not exact due to excessive penetrations; as a consequence there are oscillations in the solution. As the stabilization parameter $\tau$ reduces (or the penalty parameter $K_{N}$ increases), the two methods tend to improve the solution, reducing the fluctuations, except for the transit through the corners due to the large size of the element (see Figs. 9 and 10). Additionally the solution in terms of the gap is best maintained in the stabilized Lagrange multiplier approach for either of the cases.

However, while approaching the final part of the simulation the penalty method is unable to simulate the expansion of the block near the opening. Finally with respect to the computational efficiency of the methods, it is seen that, generically, the number of iterations is smaller in the case of the Lagrange multiplier method than in the penalty approach, except for the flat region where both exhibit a similar behavior. In particular, when the active set is predicted cor- rectly, the Lagrange multiplier takes 3 iterations to converge, whereas the regularized penalty takes about 4 iterations to achieve convergence.

In summary, we conclude that, for this problem, the Lagrange multiplier method has a clear advantage over the penalty method. In fact, the Lagrange multiplier method obtains the practically acceptable solution throughout the range considered. This advantage is also evident in terms of the gap measures, where the penalty method always has a greater penetration.

\subsubsection{Draw bead simulation}

The third example is taken from a well known simulation, [15], used in metal forming industry, where a metal sheet is deformed into the desired configuration by the punch and the die. The sheet material is considered to follow a Neo-Hookean material model with $E=2 \times 10^{5} \mathrm{MPa}$ and $v=0.1$. The die is considered made of the same type of material with $E=2 \times 10^{8} \mathrm{MPa}$ and $v=0.0$. The

(a)

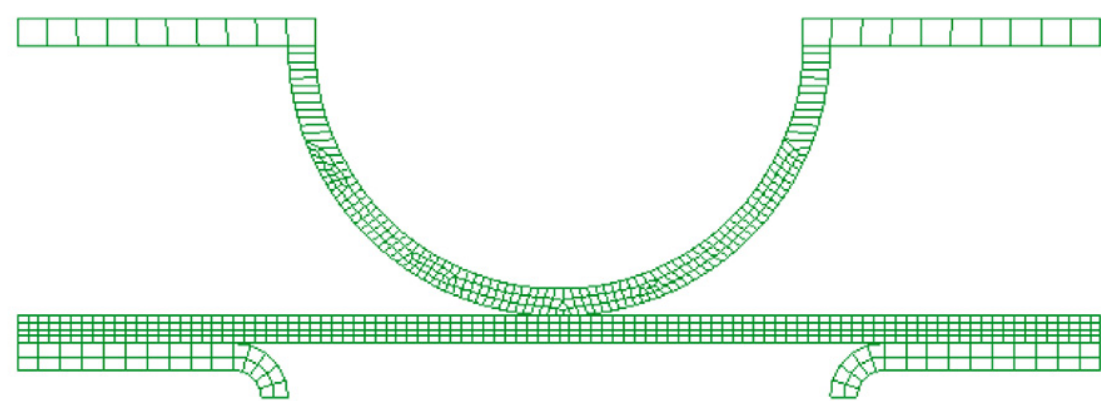

(b)

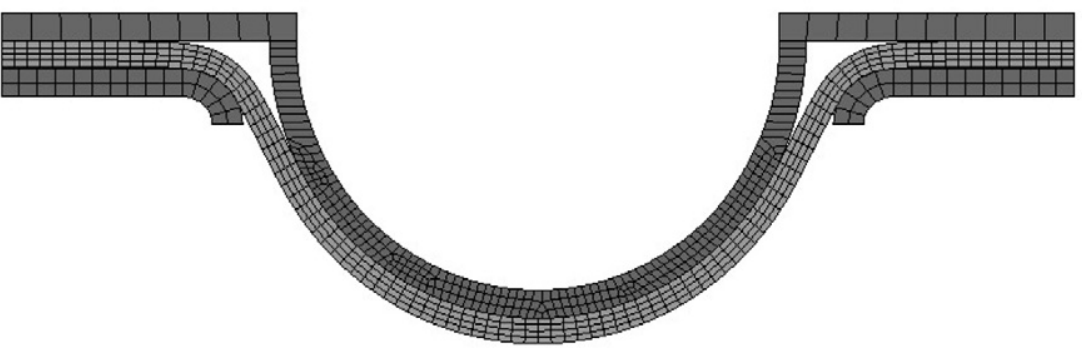

Fig. 12. Mesh geometry for the draw bead problem in: (a) undeformed state and (b) final deformed configuration.

(a)

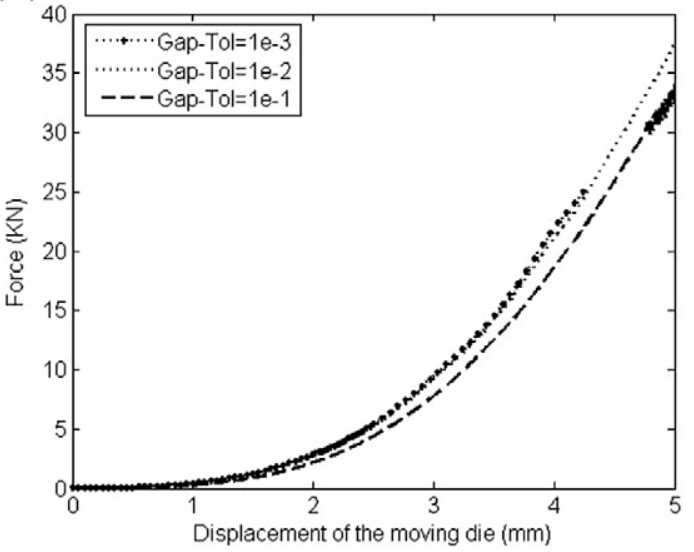

(b)

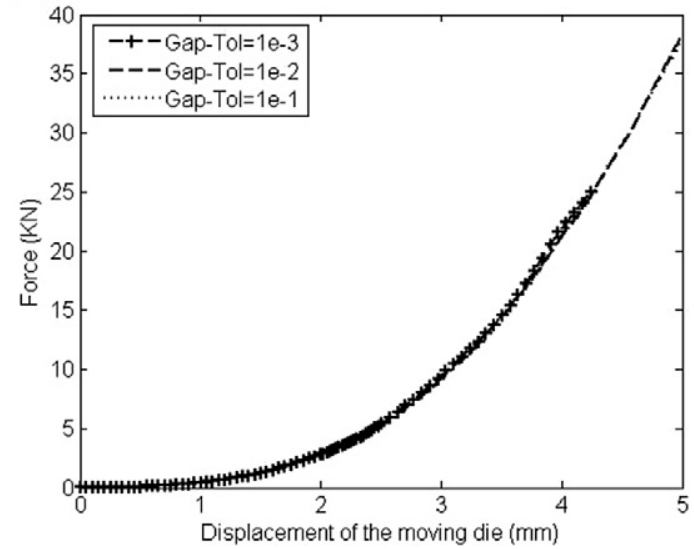

Fig. 13. Comparison of the force vs. displacement plot obtained using: (a) penalty method; (b) SLMM. 
geometry and the FE mesh in the undeformed configuration are shown in Fig. 12(a). The dimensions of the geometry of the problem are chosen as given in [15].

During the simulation, the bottom bead is held fixed, and the upper bead (or punch) is moved downwards with a total displacement of $5 \mathrm{~mm}$. Both ends of the sheet are held fixed along the $X$ direction. The final deformed configuration of the sheet is shown in Fig. 12(b).

This particular example is chosen to assess the relative performance of the Lagrange multiplier method and the penalty method in the frictional case. Hence the coefficient of friction is considered as $\mu=0.1$ and the simulation is performed using 1000 time steps.

Fig. 13(a) and (b) shows the force versus displacement plot, as the bead moves down and the reaction forces are computed along the two edges of the upper bead (where the displacements are applied), for the penalty and the Lagrange multiplier methods respectively.

In this example, the value of the allowable gap between the two contacting bodies (Gap-Tol in the figure) is supplied as the input parameter, instead of the penalty parameters. Then, the corresponding stabilization parameter (in case of SLMM) or the penalty parameters (in the penalty method) are being calculated within the code. It is seen, that it was not possible to achieve up to the final stage of deformation corresponding to a gap value of $10^{-3} \mathrm{~mm}$. In this case, the value of the stabilization parameter becomes very small after a certain time step, so that the FE solution fails to converge, in the case of SLMM, or the penalty parameter becomes too high to make the solution unstable in the case of penalty method. However, if we start increasing the gap value within the range $[0.01,0.1 \mathrm{~mm}]$ the simulation runs up to the final time step for both cases. Further it is observed, in the case of SLMM, that the computed reaction value remains exactly the same, even if the gap value changes, as seen in Fig. 13(b). However, in case of the penalty method, the reactions are getting altered substantially along with the change in the gap value, as observed in Fig. 13(a). Moreover, as the gap value is increased to $0.1 \mathrm{~mm}$ the reaction computed using the penalty method predicts much lower value compared to that of the SLMM method. Hence it can be pointed out that the solution obtained using the penalty method is much more sensitive to the change in the gap parameter value compared to that of the Lagrange multiplier method. Hence, in this particular case, the SLMM method performs more robustly in the case of frictional contact. However, for a more definitive conclusion, some more examples including friction would be needed, which has been left for future work.

\section{Concluding remarks}

The present work summarizes the performance of the contact domain method (CDM) in terms of two different implementations namely, the Lagrange multiplier based implementation and the penalty strategy. In particular the treatment of the constraints using the penalty method has been derived, while the Lagrange multiplier based formulation is summarized from [14].

Emphasis has been given on pointing out the equivalence between the two strategies, and mathematically it has been shown that indeed a simple relation can be established between the duo, through the penalty parameter of the penalty strategy and the stabilization parameter of the Lagrange multiplier method $\left(K \approx \frac{1}{2 \tau}\right)$. However, due to the distinct difference in the constraint imposition, finally the two methods result into two different formulations.

Several numerical tests, including a contact patch test, have been performed to reinforce the comparison and both the methods pass the patch test with reasonable accuracy.
It is seen that in general both the methods perform reasonably well. In specific, as we increase the value of the penalty parameter $K$ (or decrease the value of the stabilization factor, $\tau$, in the Lagrange multiplier method), the two results tend to match. However, the Lagrange multiplier method shows much smaller sensitivity for the forces and better estimates the gap, in comparison with the penalty method and this characteristic confers its greater robustness near the solution. Additionally, in critical cases a clear advantage is noticed in terms of the applicability of the Lagrange multiplier method (e.g. when there is a separation or an abrupt change in the cross section between two contacting bodies), where penalty method performs badly. Finally, in terms of computational cost the Lagrange multiplier approach does not lag behind compared to the penalty method. On the contrary, unlike what is generally reported for other Lagrange multiplier based methods, where the resolution for the Lagrange multipliers computation increases the computational cost, in the proposed CDM they can be condensed out at the contact patch level and they do not contribute to increase the computational costs in front of the penalty one. Lastly, it has to be mentioned that all the conclusions drawn above are solely based on the analysis performed using the CDM theory. However, for more definitive conclusions, it is necessary to do a more detailed comparative study with the existing contact methods such as with node-to-segment formulation, which has been left for future scope of the work.

\section{Acknowledgments}

The Spanish Ministry of Science and Innovation and the Catalan Government Research Department are gratefully acknowledged for their financial support under Grants BIA2008-00411 and 2009 SGR 1510 , respectively.

\section{Appendix A. Derivation of the contact force vector and stiffness matrix for both considered methods}

The notations used in the following section are similar to the earlier development as described in [14].

\section{A.1. Force vectors using penalty approach}

With reference to [14], the variation of the current normal and tangential vectors and the gap intensities are given by:

$$
\begin{aligned}
& \delta \mathbf{n}=-(\mathbf{n} \cdot \operatorname{grad}(\delta \mathbf{u}) \cdot \mathbf{t}) \mathbf{t}=-(\mathbf{t} \otimes \mathbf{n}) \cdot \frac{\partial \delta \mathbf{u}}{\partial t}, \\
& \delta \mathbf{t}=(\mathbf{n} \cdot \operatorname{grad}(\delta \mathbf{u}) \cdot \mathbf{t}) \mathbf{n}=(\mathbf{n} \otimes \mathbf{n}) \cdot \frac{\partial \delta \mathbf{u}}{\partial t},
\end{aligned}
$$

and

$$
\begin{aligned}
& \delta \bar{g}_{N}=\bar{g}_{N} \mathbf{n} \cdot \operatorname{grad}(\delta \mathbf{u}) \cdot \mathbf{n}, \\
& \delta \bar{g}_{T}=\bar{g}_{N}(\mathbf{n} \cdot \operatorname{grad}(\delta \mathbf{u}) \cdot \mathbf{t}+\mathbf{t} \cdot \operatorname{grad}(\delta \mathbf{u}) \cdot \mathbf{n})+\bar{g}_{T} \mathbf{t} \cdot \operatorname{grad}(\delta \mathbf{u}) \cdot \mathbf{t},
\end{aligned}
$$

respectively. Now starting with Eq. (39), contribution corresponding to normal contact is given as:

$$
\begin{aligned}
\delta \Pi_{\text {cont }}^{(N)} & =\int_{D^{(n)}} \tilde{t}_{N} \delta \bar{g}_{N} d D \\
& =\int_{D^{(n)}} \tilde{t}_{N} \bar{g}_{N} \mathbf{n} \cdot \operatorname{grad}(\delta \mathbf{u}) \cdot \mathbf{n} d D ;
\end{aligned}
$$

Considering the fact that all the quantities appearing in the integrand namely, $t_{N}, \bar{g}_{N}, \mathbf{n}$ and $\operatorname{grad}(\delta \mathbf{u})$ are constant for a linear triangular patch, the integration results into, 
$\delta \Pi_{\text {cont }}^{(N)}=\frac{1}{2} L H \tilde{t}_{N} \bar{g}_{N} \mathbf{n} \cdot \operatorname{grad}(\delta \mathbf{u}) \cdot \mathbf{n}$.

Using Eq. (40) in (68) we obtain,

$\delta \Pi_{\text {cont }}^{(N)}=\frac{1}{2} L H K_{N}^{*} \bar{g}_{N}^{2} \mathbf{n} \cdot \operatorname{grad}(\delta \mathbf{u}) \cdot \mathbf{n}$.

By replacing $\bar{g}_{N}=\frac{g_{N}^{0(3)}}{g_{N}^{(3)}}=\frac{H}{h}$ and $K_{N}^{*}=H K_{N}$, Eq. (93) can be inserted into Eq. (69) and, after some simplications, the normal contact force vector is given by,

$F_{\text {cont }}^{(N)}=\frac{1}{2} \operatorname{LhK} \mathbf{n} \cdot \sum_{I=1}^{3}\left(h \frac{\partial N_{I}}{\partial n}\right)$.

Similarly, the virtual work corresponding to a virtual tangential stick is given as:

$$
\begin{aligned}
\delta \Pi_{\text {cont }}^{(T)}= & \int_{D^{(T)}} \tilde{t}_{T} \delta \bar{g}_{T} d D=\int_{D^{(T)}} \tilde{t}_{T}\left(\bar{g}_{N} \mathbf{n} \cdot \operatorname{grad}(\delta \mathbf{u}) \cdot \mathbf{n}\right. \\
& \left.+\mathbf{n} \cdot G R A D(\delta \mathbf{u}) \cdot \operatorname{Nsign}\left(g_{N}^{0}\right)\right) d D \text { [see Ref. [5]]. }
\end{aligned}
$$

For a triangular patch this can be simplified as,

$\delta \Pi_{\text {cont }}^{(T)}=\frac{L H}{2} \tilde{t}_{T}\left(\bar{g}_{N} \mathbf{n} \cdot \operatorname{grad}(\delta \mathbf{u}) \cdot \mathbf{n}\right.$

$\left.+\mathbf{t} \cdot \operatorname{GRAD}(\delta \mathbf{u}) \cdot \mathbf{N} \operatorname{sign}\left(g_{N}^{0}\right)\right) d D$ [see Ref. [5]]

replacing $\tilde{t}_{T}$ with Eq. (49) and using $\bar{g}_{N}=\frac{H}{h}$, one can obtain,

$\delta \Pi_{\text {cont }}^{(T)}=\frac{L H}{2}\left(\tilde{t}_{T, n}+K_{T}^{*} \bar{g}_{T}\right)\left(\frac{h}{H} \mathbf{n} \cdot \operatorname{grad}(\delta \mathbf{u}) \cdot \mathbf{t}+\mathbf{t} \cdot G R A D(\delta \mathbf{u}) \cdot \mathbf{N} \operatorname{sign}\left(g_{N}^{0}\right)\right) d D$

and further simplification results into,

$\delta \Pi_{\text {cont }}^{(T)}=\frac{L}{2}\left(\tilde{t}_{T, n}+K_{T}^{*} \bar{g}_{T}\right)\left[h\left(\mathbf{n} \cdot \sum_{I=1}^{3} \frac{\partial N_{I}}{\partial t} \delta \mathbf{u}_{I}\right)+\left(\mathbf{t} \cdot \sum_{I=1}^{3} H \frac{\partial N_{I}}{\partial N} \delta \mathbf{u}_{I}\right)\right] \forall \delta \mathbf{u}_{I}$.

Hence the stick force vector is given by,

$F_{\text {cont }}^{T}=\frac{1}{2} L\left(\tilde{t}_{T, n}+K_{T}^{*} \bar{g}_{T}\right)\left[h\left(\mathbf{n} \cdot \sum_{I=1}^{3}\left(\frac{\partial N_{I}}{\partial t}\right)\right)+\left(\mathbf{t} \cdot \sum_{I=1}^{3} H \frac{\partial N_{I}}{\partial N}\right)\right]$.

Now for the slip part, the virtual work is of the form:

$\delta \Pi_{\text {cont }}^{(\text {slip })}=\int_{D^{(N)} / D^{(T)}} \tilde{\mathcal{T}} \delta \bar{g}_{T} d D=\frac{L H}{2} \tilde{\mathcal{T}} \delta \bar{g}_{T}$.

Putting back the expression for $\delta \bar{g}_{T}$ and simplifying as earlier, one could get,

$\delta \Pi_{\text {cont }}^{(\text {slip }}=\frac{L}{2} \widetilde{\mathcal{T}}\left[h\left(\mathbf{n} \cdot \sum_{I=1}^{3} \frac{\partial N_{I}}{\partial t} \delta \mathbf{u}_{I}\right)+\left(\mathbf{t} \cdot \sum_{I=1}^{3} H \frac{\partial N_{I}}{\partial N} \delta \mathbf{u}_{I}\right)\right] \quad \forall \delta \mathbf{u}_{I}$.

Hence, the slip force vector is given by,

$F_{\text {cont }}^{T}=\frac{1}{2} L \widetilde{\mathcal{T}}\left[h\left(\mathbf{n} \cdot \sum_{I=1}^{3}\left(\frac{\partial N_{I}}{\partial t}\right)\right)+\left(\mathbf{t} \cdot \sum_{I=1}^{3} H \frac{\partial N_{I}}{\partial N}\right)\right]$,

where $\tilde{\mathcal{T}}$ has to be evaluated using Eq. $(54)_{2}$.

\section{A.2. Force vectors using Lagrange multiplier method}

Considering the virtual work term corresponding to normal contact, we obtain

$\delta \Pi_{\text {cont }}^{(N)}=\int_{D^{N}} \lambda_{N} \delta \bar{g}_{N} d D$.
Further, considering that all terms appearing in the integrand are constant for a linear triangular patch, the integration results into:

$\delta \Pi_{\text {cont }}^{(N)}=\frac{1}{2} L H \Lambda_{N} \bar{g}_{N} \mathbf{n} \cdot \operatorname{grad}(\delta \mathbf{u}) \cdot \mathbf{n}$.

By putting the expression for $\bar{g}_{N}$ the normal force component is given by,

$F_{\text {cont }}^{N}=\frac{1}{2} L \Lambda_{N} \mathbf{n} \cdot \sum_{I=1}^{3}\left(h \frac{\partial N_{I}}{\partial n}\right)$

Similarly, the tangential stick component can be obtained as:

$\delta \Pi_{\text {cont }}^{(T)}=\int_{D^{T}} \lambda_{T} \delta \bar{g}_{T} d D$.

Integrating as [14] for a triangular patch yields the force component as,

$F_{\text {cont }}^{T}=\frac{1}{2} L \Lambda_{T}\left[h \mathbf{n} \cdot \sum_{I=1}^{3} \frac{\partial N_{I}}{\partial t}+\mathbf{t} \cdot \sum_{I=1}^{3}\left(H \frac{\partial N_{I}}{\partial N}\right)\right]$.

For the tangential slip part, considering the virtual work corresponding to a virtual slip of $\delta \bar{g}_{T}$ as:

$\delta \Pi_{\text {cont }}^{(N \backslash T)}=\int_{D^{N \backslash T}} \mathcal{T} \delta \bar{g}_{T} d D=\frac{1}{2} L H \mathcal{T} \delta \bar{g}_{T}\left[\right.$ where $\left.\mathcal{T}=\mu \operatorname{sign}\left(\bar{g}_{T}\right)\left|\Lambda_{N}\right|\right]$.

Using similar simplification as done in stick case, the force component can be obtained as:

$F_{\text {cont }}^{\text {slip }}=\frac{1}{2} L \mathcal{T}\left[h \mathbf{n} \cdot \sum_{I=1}^{3} \frac{\partial N_{I}}{\partial t}+\mathbf{t} \cdot \sum_{I=1}^{3}\left(H \frac{\partial N_{I}}{\partial N}\right)\right]$.

\section{A.3. Stiffness matrices using the penalty approach}

Considering the work corresponding to normal contact, one could get,

$\Delta \delta \Pi_{\text {cont }}^{(N)}=\frac{L H}{2}\left(\Delta \tilde{t}_{N} \delta \bar{g}_{N}+\tilde{t}_{N} \Delta \delta \bar{g}_{N}\right)$.

Using Eq. (43) and $\delta \bar{g}_{N}=\bar{g}_{N} \mathbf{n} \cdot \operatorname{grad}(\delta \mathbf{u}) \cdot \mathbf{n}$ (as derived in [14]) and putting $\bar{g}_{N}=\frac{H}{h}$, and $K_{N}^{*}=H K_{N}$, it can be rewritten as:

$$
\begin{aligned}
\Delta \delta \Pi_{\text {cont }}^{(N)}=[K]= & \frac{L}{2} K_{N}\left[\left(\mathbf{n} \cdot h \frac{\partial \Delta \mathbf{u}}{\partial n}\right)\left(\mathbf{n} \cdot h \frac{\partial \delta \mathbf{u}}{\partial n}\right)-h\left(\mathbf{n} \cdot \frac{\partial \Delta \mathbf{u}}{\partial t}\right)\left(\mathbf{t} \cdot h \frac{\partial \delta \mathbf{u}}{\partial n}\right)-\right. \\
& \left.-h\left(\mathbf{t} \cdot h \frac{\partial \Delta \mathbf{u}}{\partial n}\right)\left(\mathbf{n} \cdot \frac{\partial \delta \mathbf{u}}{\partial t}\right)-h^{2}\left(\mathbf{n} \cdot \frac{\partial \Delta \mathbf{u}}{\partial t}\right)\left(\mathbf{n} \cdot \frac{\partial \delta \mathbf{u}}{\partial t}\right)\right] .
\end{aligned}
$$

Hence the stiffness matrix in component form can be written as:

$$
\begin{aligned}
K_{i p j q} & =\frac{L}{2} K_{N}\left[\left(n_{i} h \frac{\partial N_{P}}{\partial n}\right)\left(n_{j} h \frac{\partial N_{Q}}{\partial n}\right)-h\left(t_{i} h \frac{\partial N_{P}}{\partial n}\right)\left(n_{j} \frac{\partial N_{Q}}{\partial n}\right)\right. \\
& \left.=-h\left(n_{i} \frac{\partial N_{P}}{\partial n}\right)\left(t_{j} h \frac{\partial N_{Q}}{\partial n}\right)-h^{2}\left(n_{i} \frac{\partial N_{P}}{\partial n}\right)\left(n_{j} \frac{\partial N_{Q}}{\partial n}\right)\right] .
\end{aligned}
$$

Similarly, the component of the virtual work corresponding to the stick part can be taken as:

$\Delta \delta \Pi_{\text {cont }}^{(T)}=\frac{L H}{2}\left(\Delta \tilde{t}_{T} \delta \bar{g}_{T}+\tilde{t}_{T} \Delta \delta \bar{g}_{T}\right)$.

As we know,

$$
\begin{aligned}
\tilde{t}_{T, n+1}= & \tilde{t}_{T, n}+K_{T}^{*} \bar{g}_{T} \Rightarrow \Delta \tilde{t}_{T}=K_{T}^{*} \Delta \bar{g}_{T} ; \quad \text { with } \Delta \bar{g}_{T} \\
= & \bar{g}_{N} \mathbf{n} \cdot \operatorname{grad}(\Delta \mathbf{u}) \cdot \mathbf{t}+\bar{g}_{N} \mathbf{t} \cdot \operatorname{grad}(\Delta \mathbf{u}) \cdot \mathbf{n}+\bar{g}_{T} \mathbf{t} \\
& \cdot \operatorname{grad}(\Delta \mathbf{u}) \cdot \mathbf{t}
\end{aligned}
$$


and

$$
\begin{aligned}
\Delta \delta \bar{g}_{T}= & \bar{g}_{N}\left(\mathbf{n} \cdot \frac{\partial \delta \mathbf{u}}{\partial t}\right)\left(\mathbf{n} \cdot \frac{\partial \Delta \mathbf{u}}{\partial t}\right)-\bar{g}_{N}\left(\mathbf{t} \cdot \frac{\partial \delta \mathbf{u}}{\partial t}\right)\left(\mathbf{n} \cdot \frac{\partial \Delta \mathbf{u}}{\partial t}\right) \\
& -\bar{g}_{N}\left(\mathbf{n} \cdot \frac{\partial \delta \mathbf{u}}{\partial t}\right)\left(\mathbf{t} \cdot \frac{\partial \Delta \mathbf{u}}{\partial t}\right)+\bar{g}_{N}\left(\mathbf{n} \cdot \frac{\partial \delta \mathbf{u}}{\partial n}\right)\left(\mathbf{n} \cdot \frac{\partial \Delta \mathbf{u}}{\partial t}\right) \\
& +\bar{g}_{T}\left(\mathbf{n} \cdot \frac{\partial \delta \mathbf{u}}{\partial t}\right)\left(\mathbf{n} \cdot \frac{\partial \Delta \mathbf{u}}{\partial t}\right)
\end{aligned}
$$

as explained in [14]. Using the above formula, and the expressions for $K_{T}^{*}$ and $\bar{g}_{N}$, Eq. (89) can be re-written as:

$$
\begin{aligned}
\Delta \delta \Pi_{\text {cont }}^{(T)}= & \frac{L}{2} K_{T}\left[h\left(\mathbf{n} \cdot \frac{\partial \Delta \mathbf{u}}{\partial t}\right)+\left(\mathbf{t} \cdot H \frac{\partial \Delta \mathbf{u}}{\partial N}\right)\right]\left[h\left(\mathbf{n} \cdot \frac{\partial \delta \mathbf{u}}{\partial t}\right)+\left(\mathbf{t} \cdot H \frac{\partial \delta \mathbf{u}}{\partial N}\right)\right] \\
& +\frac{L^{2}}{2} \tilde{t}_{T}\left[\left(\mathbf{n} \cdot h \frac{\partial \Delta \mathbf{u}}{\partial n}\right)\left(\mathbf{n} \cdot \frac{\partial \delta \mathbf{u}}{\partial t}\right)-h\left(\mathbf{n} \cdot \frac{\partial \Delta \mathbf{u}}{\partial t}\right)\left(t \cdot \frac{\partial \delta \mathbf{u}}{\partial t}\right)\right. \\
& \left.-h\left(\mathbf{t} \cdot \frac{\partial \Delta \mathbf{u}}{\partial t}\right)\left(\mathbf{n} \cdot \frac{\partial \delta \mathbf{u}}{\partial t}\right)+\left(\mathbf{n} \cdot \frac{\partial \Delta \mathbf{u}}{\partial t}\right)\left(\mathbf{n} \cdot H \frac{\partial \Delta \mathbf{u}}{\partial N}\right)\right] .
\end{aligned}
$$

Finally, the stiffness matrix in component form is given as:

$$
\begin{aligned}
K_{i p j q}^{T}= & \frac{1}{2} L K_{T}\left[h\left(n_{i} \frac{\partial N_{P}}{\partial t}\right)+\left(t_{i} H \frac{\partial N_{P}}{\partial N}\right)\right]\left[h\left(n_{j} \frac{\partial N_{q}}{\partial t}\right)+\left(t_{i} H \frac{\partial N_{q}}{\partial N}\right)\right] \\
& +\frac{1}{2} L \tilde{t}_{T}\left[\left(n_{i} \frac{\partial N_{P}}{\partial t}\right)\left(n_{j} h \frac{\partial N_{Q}}{\partial n}\right)-h\left(t_{i} \frac{\partial N_{P}}{\partial t}\right)\left(n_{j} \frac{\partial N_{Q}}{\partial t}\right)\right. \\
& \left.-h\left(n_{i} \frac{\partial N_{P}}{\partial t}\right)\left(t_{j} \frac{\partial N_{Q}}{\partial t}\right)+\left(n_{i} H \frac{\partial N_{P}}{\partial N}\right)\left(n_{j} \frac{\partial N_{Q}}{\partial t}\right)\right] .
\end{aligned}
$$

To obtain the stiffness matrix corresponding to tangential slip, let us consider,

$\Delta \delta \Pi_{\text {cont }}^{(\text {slip })}=\frac{L H}{2}\left(\Delta \widetilde{\mathcal{T}} \delta \bar{g}_{T}+\tilde{\mathcal{T}} \Delta \delta \bar{g}_{T}\right)$.

As $\tilde{\mathcal{T}}=\mu \operatorname{sign}\left(t_{T}^{T R}\right)\left|t_{N}\right|$,

$\Rightarrow \Delta \widetilde{\mathcal{T}}=\mu \operatorname{sign}\left(t_{T}^{T R}\right) \operatorname{sign}\left(t_{N}\right) \Delta t_{N} ; \quad$ with $\Delta t_{N}=K_{N}^{*} \Delta \bar{g}_{N}$

Further simplification using $K_{N}^{*}=H K_{N}$ allows us to write,

$$
\begin{aligned}
\Delta \delta \Pi_{\text {cont }}^{(\text {slip })}= & \frac{L}{2}\left\{\mu \operatorname { s i g n } ( t _ { T } ^ { T R } ) \operatorname { s i g n } ( t _ { N } ) K _ { N } ( \mathbf { n } \cdot h \frac { \partial \Delta \mathbf { u } } { \partial n } ) \left[h\left(\mathbf{n} \cdot \frac{\partial \delta \mathbf{u}}{\partial t}\right)\right.\right. \\
& \left.+\left(\mathbf{t} \cdot H \frac{\partial \delta \mathbf{u}}{\partial N}\right)\right]+\widetilde{\mathcal{T}}\left[\left(\mathbf{n} \cdot \frac{\partial \delta \mathbf{u}}{\partial t}\right)\left(\mathbf{n} \cdot h \frac{\partial \Delta \mathbf{u}}{\partial n}\right)\right. \\
& -h\left(\mathbf{t} \cdot \frac{\partial \delta \mathbf{u}}{\partial t}\right)\left(\mathbf{n} \cdot \frac{\partial \Delta \mathbf{u}}{\partial t}\right)-h\left(\mathbf{n} \cdot \frac{\partial \delta \mathbf{u}}{\partial t}\right)\left(\mathbf{t} \cdot \frac{\partial \Delta \mathbf{u}}{\partial t}\right) \\
& \left.\left.+\left(\mathbf{n} \cdot H \frac{\partial \delta \mathbf{u}}{\partial N}\right)\left(\mathbf{n} \cdot \frac{\partial \Delta \mathbf{u}}{\partial t}\right)\right]\right\} .
\end{aligned}
$$

And finally the stiffness matrix in the component form looks like,

$$
\begin{aligned}
K_{\text {ipjq }}^{\text {slip }}= & \frac{1}{2} L\left\{\mu \operatorname{sign}\left(\tilde{t}_{T}^{T R}\right) \operatorname{sign}\left(\tilde{t}_{N}\right) K_{N}\left[h\left(n_{i} \frac{\partial N_{P}}{\partial t}\right)+\left(t_{i} H \frac{\partial N_{P}}{\partial N}\right)\right]\left(n_{j} h \frac{\partial N_{q}}{\partial n}\right)\right. \\
& +\widetilde{\mathcal{T}}\left[\left(n_{i} \frac{\partial N_{P}}{\partial t}\right)\left(n_{j} h \frac{\partial N_{q}}{\partial n}\right)-h\left(t_{i} \frac{\partial N_{P}}{\partial t}\right)\left(n_{j} \frac{\partial N_{q}}{\partial t}\right)\right. \\
& \left.\left.-h\left(n_{i} \frac{\partial N_{P}}{\partial t}\right)\left(t_{j} \frac{\partial N_{q}}{\partial t}\right)+\left(n_{i} H \frac{\partial N_{P}}{\partial N}\right)\left(n_{j} \frac{\partial N_{q}}{\partial t}\right)\right]\right\} .
\end{aligned}
$$

\section{A.4. Stiffness matrices using the Lagrange multiplier method}

To obtain the normal component, let us consider the variation of the normal contact as,

$\Delta \delta \Pi_{\text {cont }}^{N}=\frac{L H}{2}\left(\Delta \Lambda_{N} \delta \bar{g}_{N}+\Lambda_{N} \Delta \delta \bar{g}_{N}\right)$.

To compute the variation for the Lagrange multiplier see [14]. By replacing the expressions for $\Delta \mathbf{n}, \delta \bar{g}_{N}$ and $\Delta \delta \bar{g}_{N}$ as given in [14], Eq. (98) can be re-written as:
$\Delta \delta \Pi_{\text {cont }}^{N}=\frac{L H}{2}[\underbrace{-t_{T}(\mathbf{n} \cdot \operatorname{grad}(\Delta \mathbf{u}) \cdot \mathbf{t}) \delta \bar{g}_{N}+\frac{H}{2 \tau} \Delta \bar{g}_{N} \delta \bar{g}_{N}}_{K_{I}}+\underbrace{\mathbf{n} \cdot \Delta \mathbf{P} \cdot \mathbf{N} \delta \bar{g}_{N}}_{K_{I I}}]$.

Now, from the 1 st term using $\bar{g}_{N}=\frac{h}{H}$, in the component form, the stiffness matrix can be written as:

$$
\begin{aligned}
{\left[K_{I}\right]_{i p j q}=} & \frac{L}{2}\left[-t_{T}\left(\mathbf{n}_{i} h \frac{\partial \Delta \mathbf{u}}{\partial t}\right)\left(\mathbf{n}_{j} \cdot \frac{\partial \delta \mathbf{u}}{\partial n}\right)+\frac{1}{2 \tau}\left(\mathbf{n}_{i} h \frac{\partial \Delta \mathbf{u}}{\partial n}\right)\left(\mathbf{n}_{j} h \frac{\partial \delta \mathbf{u}}{\partial n}\right)\right. \\
& -\Lambda_{N}\left(\mathbf{t}_{i} h \frac{\partial \delta \mathbf{u}}{\partial n}\right)\left(\mathbf{n}_{j} \frac{\partial \Delta \mathbf{u}}{\partial t}\right)-\Lambda_{N}\left(\mathbf{n}_{i} \frac{\partial \delta \mathbf{u}}{\partial t}\right)\left(\mathbf{t}_{j} h \frac{\partial \Delta \mathbf{u}}{\partial n}\right) \\
& \left.-\Lambda_{N} h\left(\mathbf{n}_{i} \frac{\partial \delta \mathbf{u}}{\partial t}\right)\left(\mathbf{n}_{j} \frac{\partial \Delta \mathbf{u}}{\partial t}\right)\right] .
\end{aligned}
$$

For the 2nd term, let us start with the relation between 1st P-K stress and 2nd $\mathrm{P}-\mathrm{K}$ stress as:

$\mathbf{P}=\mathbf{f} \cdot \mathbf{S} \Rightarrow \Delta \mathbf{P}=\Delta \mathbf{f} \cdot \mathbf{S}+\mathbf{f} \cdot \Delta \mathbf{S}$

In the component form,

$\left[K_{I I}\right]_{i p j q}=\frac{L}{2}\left(n_{i} h \frac{\partial N_{P}}{\partial n}\right)\left(n_{K} \Delta \mathbf{P}_{k l q j} N_{l}\right)$.

For the detail derivation please refer to [14].

For the tangential stick component, let us take the virtual work expression as

$\Delta \delta \Pi_{\text {cont }}^{T}$

$=\frac{L H}{2}[\underbrace{t_{N}(\mathbf{n} \cdot \operatorname{grad}(\Delta \mathbf{u}) \cdot \mathbf{t}) \delta \bar{g}_{T}+\frac{H}{2 \tau} \Delta \bar{g}_{T} \delta \bar{g}_{T}+\Lambda_{T} \Delta \delta \bar{g}_{T}}_{K_{I}}+\underbrace{\mathbf{t} \cdot \Delta \mathbf{P} \cdot \mathbf{N} \delta \bar{g}_{T}}_{K_{I I}}]$.

Using the expression for $\Delta \bar{g}_{T}, \operatorname{grad}(\Delta \mathbf{u})$ and $\Delta \delta \bar{g}_{T}$, and $\bar{g}_{N}=\frac{h}{H}$, in the component form, the 1 st term of the stiffness matrix reads,

$$
\begin{aligned}
{\left[K_{I}\right]_{\hat{i} j q}=} & \frac{L}{2}\left\{t_{N}\left[h\left(n_{i} \frac{\partial N_{P}}{\partial t}\right)+\left(t_{i} H \frac{\partial N_{P}}{\partial N}\right)\right]\left(n_{j} \frac{\partial N_{q}}{\partial t}\right)\right. \\
& +\frac{1}{2 \tau}\left[h\left(n_{i} \frac{\partial N_{P}}{\partial t}\right)+\left(t_{i} H \frac{\partial N_{P}}{\partial N}\right)\right]\left[h\left(n_{j} \frac{\partial N_{q}}{\partial t}\right)+\left(t_{i} H \frac{\partial N_{q}}{\partial N}\right)\right] \\
& +\Lambda_{T}\left[\left(n_{i} \frac{\partial N_{P}}{\partial t}\right)\left(n_{j} h \frac{\partial N_{q}}{\partial n}\right)-h\left(t_{i} \frac{\partial N_{P}}{\partial t}\right)\left(n_{j} \frac{\partial N_{q}}{\partial t}\right)\right. \\
& \left.\left.-h\left(n_{i} \frac{\partial N_{P}}{\partial t}\right)\left(t_{j} \frac{\partial N_{q}}{\partial t}\right)+\left(n_{i} H \frac{\partial N_{P}}{\partial N}\right)\left(n_{j} \frac{\partial N_{q}}{\partial t}\right)\right]\right\}
\end{aligned}
$$

and the 2nd term in the component form is given as,

$\left[K_{I I}\right]_{i p j q}=\frac{L}{2}\left[h\left(n_{i} \frac{\partial N_{p}}{\partial t}\right)+\left(t_{i} H \frac{\partial N_{p}}{\partial N}\right)\right]\left(t_{K} \Delta P_{k l q j} N_{l}\right)$

Finally, the stiffness component for tangential slip part can be obtained as follows:

$$
\begin{aligned}
\Delta \delta \Pi_{\text {cont }}^{\text {slip }}= & \frac{L H}{2}\left[-\mu \operatorname{sign}\left(\bar{g}_{T}\right) \operatorname{sign}\left(\Lambda_{N}\right) t_{T}(\mathbf{n} \cdot \operatorname{grad}(\Delta \mathbf{u}) \cdot \mathbf{t}) \delta \bar{g}_{T}\right. \\
& +\mu \operatorname{sign}\left(\bar{g}_{T}\right) \operatorname{sign}\left(\Lambda_{N}\right) \frac{H}{2 \tau} \Delta \bar{g}_{N} \delta \bar{g}_{T}+\mathcal{T} \Delta \delta \bar{g}_{T} \\
& \left.\left.+\mu \operatorname{sign}\left(\overline{\boldsymbol{g}}_{T}\right) \operatorname{sign}\left(\Lambda_{N}\right) \mathbf{n} \cdot \Delta \mathbf{P} \cdot \mathbf{N} \cdot \delta \overline{\boldsymbol{g}}_{T}\right]\right\} K_{I I} .
\end{aligned}
$$

Using $\bar{g}_{N}=\frac{h}{H}$, in the component form, the 1 st term of the stiffness matrix can be rewritten as: 


$$
\begin{aligned}
{\left[K_{I}\right]_{i p j q}=} & \frac{L}{2}\left\{-\mu \operatorname{sign}\left(\bar{g}_{T}\right) \operatorname{sign}\left(\Lambda_{N}\right) t_{T}\left[h\left(n_{i} \frac{\partial N_{P}}{\partial t}\right)+\left(t_{i} H \frac{\partial N_{P}}{\partial N}\right)\right]\left(n_{j} \frac{\partial N_{q}}{\partial t}\right)\right. \\
& +\mu \operatorname{sign}\left(\bar{g}_{T}\right) \operatorname{sign}\left(\Lambda_{N}\right) \frac{1}{2 \tau}\left[h\left(n_{i} \frac{\partial N_{P}}{\partial t}\right)+\left(t_{i} H \frac{\partial N_{P}}{\partial N}\right)\right]\left(n_{j} h \frac{\partial N_{q}}{\partial t}\right) \\
& +\mathcal{T}\left[\bar{g}_{N}\left(n_{i} \frac{\partial N_{P}}{\partial t}\right)\left(n_{j} h \frac{\partial N_{q}}{\partial n}\right)-h\left(t_{i} \frac{\partial N_{P}}{\partial t}\right)\left(n_{j} \frac{\partial N_{q}}{\partial t}\right)\right. \\
& \left.\left.-h\left(n_{i} \frac{\partial N_{P}}{\partial t}\right)\left(t_{j} \frac{\partial N_{q}}{\partial t}\right)+\left(n_{i} H \frac{\partial N_{P}}{\partial N}\right)\left(n_{j} \frac{\partial N_{q}}{\partial t}\right)\right]\right\}
\end{aligned}
$$

and similar to the earlier, the 2nd part can be written as in the component form:

$$
\begin{aligned}
{\left[K_{I I}\right]_{i p j q}=} & \mu \operatorname{sign}\left(\bar{g}_{T}\right) \operatorname{sign}\left(\Lambda_{N}\right) \frac{L}{2}\left[h\left(n_{i} \frac{\partial N_{P}}{\partial t}\right)+\left(t_{i} H \frac{\partial N_{P}}{\partial N}\right)\right]\left(n_{K}\right. \\
& \left.\cdot \Delta P_{\text {klqj }} \cdot N_{l}\right) .
\end{aligned}
$$

\section{References}

[1] M. Asghar, P. Lyons, The contact patch test, in: International Conference on Computational Contact Mechanics, Lecce, Italy, Oral Presentation, 2009.

[2] K.J. Bathe, A.B. Chaudhary, A solution method for planar and axisymmetric contact problems, Int. J. Numer. Methods Engrg. 21 (1985) 65-88.

[3] C. Bernardi, Y. Maday, A. Patera, Domain decomposition by the mortar element method, in: H. Kasper, M. Garby (Eds.), Asymptotic and Numerical Methods for Partial Differential Equations with Critical Parameters, Kluwer Academic Publisher, Dordrecht, Netherlands, 1993, pp. 269-286.

[4] P.R. Dawson, D. Boyce, G. Eggert, A. Bradon, A consistent penalty method for contact between a deforming viscoplastic workpiece and a rigid tool, Int. J. Numer. Methods Engrg. 38 (1995) 3969-3987.

[5] K.A. Fischer, P. Wriggers, Frictionless 2D contact formulations for finite deformations based on the mortar method, Comput. Mech. 36 (2005) 226-244.

[6] K.A. Fischer, P. Wriggers, Mortar based frictional contact formulation for highe order interpolations using the moving friction cone, Comput. Methods Appl. Mech. Engrg. 195 (2006) 5020-5036.

[7] J. Hallquist, G. Goudreau, D. Benson, Sliding interfaces with contact-impact in large-scale Lagrangian computations, Comput. Methods Appl. Mech. Engrg. 51 (1985) 107-137.

[8] S. Hartmann, Kontaktanalyse dünnwandiger Strukturen bei großen Deformationen, Ph.D. Thesis, Report No. 49, Institut für Baustatik, Universität Stuttgart, Germany, 2007.

[9] S. Hartmann, J. Oliver, R. Weyler, J.C. Cante, J. Hernández, A contact domain method for large deformation frictional contact problems. Part 2: Numerical aspects, Comput. Methods Appl. Mech. Engrg. 198 (2009) 2607-2631.
[10] S. Hartmann, E. Ramm, A mortar based contact formulation for non-linear dynamics using dual Lagrange multipliers, Finite Elem. Anal. Des. 44 (2008) 245-258.

[11] S. Hartmann, R. Weyler, J. Oliver, J.C. Cante, J. Hernández, A 3D frictionless contact domain method for large deformation problems, Comput. Model. Engrg. Sci. 55 (2010) 211-269.

[12] P. Heintz, P. Hansbo, Stabilized Lagrange multiplier method for bilateral elastic contact with friction, Comput. Methods Appl. Mech. Engrg. 195 (2006) 43234333.

[13] J. Nitsche, Über ein Variationsprinzip zur Lösung von Dirichlet-Problemen bei Verwendung von Teilräumen, die keinen Randbedingungen unterworfen sind, Abhandlungen in der Mathematik an der Universität Hamburg 36 (1971) 9-15.

[14] J. Oliver, S. Hartmann, J.C. Cante, R. Weyler, J. Hernández, A contact domain method for large deformation frictional contact problems. Part 1: Theoretical basis, Comput. Methods Appl. Mech. Engrg. 198 (2009) 2591-2606.

[15] V. Padmanabhan, T. Laursen, A framework for development of surface smoothing procedures in large deformation frictional contact analysis, Finite Elem. Anal. Des. 37 (2001) 173-198.

[16] P. Papadopoulos, R. Taylor, A mixed formulation for the finite element solution of contact problems, Comput. Methods Appl. Mech. Engrg. 94 (1992) 373-389.

[17] M. Puso, T. Laursen, A mortar segment-to-segment contact method for large deformation solid mechanics, Comput. Methods Appl. Mech. Engrg. 193 (2004) 601-629.

[18] M. Puso, T. Laursen, A mortar segment-to-segment frictional contact method for large deformations, Comput. Methods Appl. Mech. Engrg. 193 (2004) 48914913.

[19] J.C. Simo, P. Wriggers, R.L. Taylor, A perturbed Lagrangian formulation for the finite element solution of contact problems, Comput. Methods Appl. Mech. Engrg. 50 (1985) 163-180.

[20] M. Tur, F.J. Fuenmayor, P. Wriggers, A mortar based frictional contact formulation for large deformations using Lagrange multipliers, Comput. Methods Appl. Mech. Engrg. 198 (2009) 2860-2873.

[21] A.L. Van, T.T.H. Nguyen, A weighted residual relationship for the contact problem with Coulomb friction, Comput. Struct. 87 (2009) 1580-1601.

[22] P. Wriggers, Computational Contact Mechanics, Wiley, 2002.

[23] P. Wriggers, J.C. Simo, A note on tangent stiffness for fully nonlinear contact problems, Commun. Appl. Numer. Methods 1 (1985) 199-203.

[24] P. Wriggers, G. Zavarise, A formulation for frictionless contact problems using a weak form introduced by Nitsche, Comput. Mech. 41 (2008) 407-420.

[25] B. Yang, T. Laursen, X. Meng, Two dimensional mortar contact methods for large deformation frictional sliding, Int. J. Numer. Methods Engrg. 62 (2005) 1183-1225.

[26] G. Zavarise, L. De Lorenzis, The node-to-segment algorithm for 2D frictionless contact: classical formulation and special cases, Comput. Methods Appl. Mech. Engrg. 198 (2009) 3428-3451.

[27] G. Zavarise, P. Wriggers, A superlinear convergent augmented Lagrangian procedure for contact problems, Engrg. Comput. 16 (1999) 88-119. 